\title{
DIE DEUTSCHE SEKTION IN PARIS
}

Warum in einem Kommuneband die Geschichte einer „,vergessenen” Pariser Sektion der Internationalen Arbeiterassoziation behandeln, die schon im August 1870 aufhörte zu existieren?

Ein Einzelbrief in deutscher Sprache aus dem belagerten Paris, selbst wenn er direkt in die Vorgeschichte der Kommune hineinreichte und mit dem Stempel des Pariser Föderalrats der IAA versehen war (Dokument VIII), erlaubte sicherlich noch keine Antwort auf diese Frage. Fand sich aber ein späterer Brief, den der gleiche Henri Bachruch, der im September 1870 intime Kenntnis der Pariser Situation und interessierte Vertrautheit mit deutschen Parteiverhältnissen ${ }^{1}$ bewiesen hatte, als "exsecrétaire de la section allemande" unterschrieb, ${ }^{2}$ wurde man aufmerksam: Eine deutsche Sektion in Paris, deren Sekretär noch während des Krieges Kontakt mit dem Zentralkomitee der Sektionsgruppe deutscher Sprache hielt, konnte eine Teilantwort bedeuten. Zunächst stellte sich mir allerdings die Vorfrage: War diese Pariser Sektion wirklich ,vergessen” oder nur - von mir - bisher übersehen? Die Antwort bleibt zwiespältig:

Zeitgenossen registrierten die Existenz der Sektion. ${ }^{3}$ Dem deutschsozialdemokratischen Kommentator des Marx'schen Bürgerkrieg der Zwischenkriegszeit war sie bekannt, ${ }^{4}$ ebenso den kommunistischen Herausgebern der kaum noch greifbaren französischen Sammlung von

1 Dass sich Bachruch angelegentlich nach der Haltung der dissidenten bayrischen Lassalleaner erkundigt, die sich auf dem Stuttgarter Kongress im Juni der SDAP angeschlossen hatten, spricht eher für als gegen eine solche Bewertung. ${ }^{2}$ Bachruch an Marx, Antwerpen 19.VI.1871 (Marx-Engels-Nachlass D 92, Fotokopie).

3 Vgl. O. Testut, Association Internationale des Travailleurs, Lyon 1870, S. $180-181$.

4 „Leo Frankel war zwar Hauptbegründer der deutschen Sektion der Internationale in Paris, war selbst aber keineswegs ein Deutscher, sondern ein Ungar". K. Marx, Der Bürgerkrieg in Frankreich, Mit Einl. u. Anm. v. A. Conrady, Berlin 1920, S. 143 (Anmerkungen des Herausgebers). 
Kommunebriefen von $1934 .^{1}$ Seither beiläufige Erwähnungen ${ }^{2}$ und Fussnoten, ${ }^{3}$ aber auch eine Mitgliederliste, ${ }^{\mathbf{4}}$ auf der sich dann der Name Leo Frankel findet. In dessen Biographie schliesslich - verständlicherweise zugespitzt auf die Person - eine erste Skizze der Tätigkeit der Sektion. ${ }^{5}$ Was interessant wäre an dieser Sektion, die den späteren "Arbeitsminister" der Kommune in den Pariser Föderalrat delegierte, hat Magda Aranyossi dort angedeutet:

„Obwohl die Mitglieder des Vereins Ausländer waren, kämpften sie mit den französischen Arbeitern zusammen und nahmen die damit verbundenen Schwierigkeiten und das Risiko auf sich."6

Dieses Element praktischen Internationalismus, die Organisation deutscher Arbeiter in der Hauptstadt des „Bas-Empire”, anschaulich zu machen, reichte die bisherige Kenntnis allerdings kaum aus.

Der glückliche Umstand, das sich im Nachlass von Johann Philipp Becker eine Reihe von Briefen fand, die gerade für diesen Aspekt aufschlussreich waren, machte die hier vorgelegte Skizze möglich.? Sie erhielt die vorläufige Form einer Serie von Momentaufnahmen und stellt mehr Fragen, als sie Antworten gibt. Hauptgegenstand ist das subjektive Element, die Organisationsarbeit; Hauptquelle sind die Selbstzeugnisse der Organisatoren. Die Pariser Umwelt erscheint zu-

1 „Frankel, Leo [...] un des dirigeants de la section allemande”, Lettres de communards et de militants de la Ire Internationale à Marx, Engels et autres..., Présenté et rédigé par Jules Rocher, Paris 1934, S. 58f.; vgl. auch ebd., S. 20. 2 So z.B. im Zusammenhang mit Henri Bachruch bei T. Erényi, ,Vlijanie Parižskoj Kommuny na rabočee i revoljucionnoe dviženie v Vengrii", in Parižskaja Kommuna 1871 g., Bd II, Moskau 1961, S. 460 unter Bezugnahme auf Dok. V; oder Karl Marx, Friedrich Engels, Werke [weiter zitiert als: MEW], Bd 33, Berlin 1966, S. 858: „Bachruch, Henri [...] Sekretär der deutschen Sektion der IAA in Paris (1870)"; ausführlicher: E. Silberner, Moses Hess - Geschichte seines Lebens, Leiden 1966, S. 613-615.

3 Vgl. z.B. The General Council of the First International [...] Minutes [weiter zitiert als: Minutes], Band 1868-1870, Moskau o.J., S. 467, Note 298.

4 Dictionnaire Biographique du Mouvement Ouvrier Français, publié sous la direction de Jean Maitron [weiter zitiert als: DBMOF], Bd IV, Paris o.J., S. 47. 5 M. Aranyossi, Leo Frankel, Berlin 1957, S. 18-21 und passim.

6 M. Aranyossi, a.a.O., S. 19.

7 Die Originale der hier benutzten Briefe befinden sich, soweit nicht ausdrücklich anders vermerkt, im Nachlass Joh. Ph. Becker im Internationaal Instituut voor Sociale Geschiedenis. In den Zitaten wurde die Orthographie modernisiert und offensichtliche Schreibfehler stillschweigend verbessert, Wort und Silbenstand jedoch beibehalten; Abweichungen von diesem Verfahren wurden vermerkt. Aus typographischen Gründen wurde - auch im Dokumentenanhang - für $\beta$ und dessen zeitgenössische Schreibweisen einheitlich ss verwendet. 
nächst kaum anders, als durch diese Zeugnisse vermittelt. ${ }^{1}$ Der „Vorgeschichte" der Sektion wurde relativ breiter Raum eingeräumt. Nur so konnte mehr erreicht werden, als Ergänzung der Biographie des einzigen Frankel; nur so war zu zeigen, dass die kurze Existenz der Section allemande Höhepunkt und Ergebnis langjähriger, mühevoller, aber beharrlicher Versuche war, deutsche Arbeiter in Paris zu organisieren.

Es erscheint verlockend, die Rekonstruktion der Vorgeschichte der deutschen Sektion mit dem Hinweis darauf zu beginnen, dass sich seit 1865 mit Moses Hess, Karl Kaub, Nils Laurens Petersen und Victor Schily mindestens vier untereinander bekannte deutsche Kommunisten der ersten Generation gleichzeitig in Paris befanden und auch Mitglieder der IAA waren. ${ }^{2}$ Nachzuweisen, dass diese Konstellation wesentlichen Einfluss auf die Versuche, deutsche Arbeiter in Paris zu organisieren, gehabt hätte, dürfte jedoch vorläufig schwer fallen.

Karl Kaub, in London 1864-65 Mitglied des provisorischen Zentralrats der Assoziation, hat anscheinend in der zweiten Hälfte der sechziger Jahre kaum mehr getan, als seinen freundschaftlichen Kontakt mit Marx aufrecht zu erhalten. In Schilys Charakteristik, Kaub sei „kommunistischer Dilletant, hauptsächlich aber Maschinenfabrikant, dem es nicht nach Wunsch geht", ${ }^{3}$ mag persönliche Enttäuschung mitspielen; wie fern Kaub der Organisationsarbeit in Paris anscheinend stand, ist dort dennoch treffend angedeutet.

Die eigentümliche Position von Moses Hess in jenen Pariser Jahren hat sein Biograph treffend geschildert. ${ }^{4}$ Sein später Bruch mit Schweitzers Social-Demokrat hat vielleicht dazu beigetragen, dass er den Kontakt mit den Pariser Lassalleanern auch danach nicht völlig verlor. Seine vorbehaltlose Parteilichkeit als Korrespondent des Volksstaat hat dann, wie sich zeigen wird, als negativer Katalysator die Gründung

1 Eine umfassendere Studie, in der diese Einseitigkeit zu korrigieren und die hier ausgewerteten Quellen vollständig zu veröffentlichen wären, hoffe ich demnächst vorzulegen.

${ }_{2}$ Die Mitgliedschaft von Hess und Petersen im Bund der Kommunisten dürfte als gesichert gelten; Schilys Bemerkung über sein Verhältnis zu Kaub: „Seitdem nennen wir uns Herr Kaub u. Herr Schily; schöne Brüder, das!' (Schily an Becker, Paris 8.VI.1866, D II 966), könnte die Angaben über Schilys Mitgliedschaft bei Wermuth und Stieber, Die Communistenverschwörungen..., Teil II, Berlin 1854, S. 111 bestätigen und für Kaub ergänzen.

a Schily an Becker, Paris, 9.XI.1866 (D II 973).

4 E. Silberner, a.a.O., passim. 
der Sektion beeinflusst. Von aktiver Mitwirkung aber wird man kaum sprechen können.

Ungleich wichtiger jedenfalls war Victor Schily. Zwar richtete sich das Hauptinteresse des seit Jahren in Paris als Rechtsberater tätigen, aus Trier gebürtigen Advokaten vornehmlich auf das, was man die grosse Politik der Internationale nennen möchte. Seine scharfsinnigen, gelegentlich skeptisch-spöttischen Beobachtungen zum Stand der Dinge in Paris sind für die Kontrolle anderer Aussagen häufig unentbehrlich. Kaub und Hess gegenüber hatte er unzweifelhaft den Vorteil grösserer Nähe; im Gegensatz zu jenen hatte er in dem Prozess, dessen Nachzeichnung hier versucht wird, eine angebbare Funktion. Bis die deutsche Sektion 1870 für kurze Zeit diese Funktion übernehmen sollte, war Schily - nicht nur, ${ }^{1}$ aber auch - Beckers „Mann in Paris”. Als Informator, vor allem aber als jene Stelle, über die der Vertrieb der Genfer Publikationen in Paris lief, und die damit - gelegentlich mehr schlecht als recht - für eine gewisse Kontinuität internationalistischer Propaganda in deutscher Sprache sorgte.

Bleibt der einzige Arbeiter in diesem Kreise, der dänische Kürschner Nils Laurens Petersen, der - gleichsam als Monument der Kontinuität wohl an allen Organisationen deutscher Arbeiter seit Weitling teilgenommen hatte. Mit ihm die Vorgeschichte der Sektion beginnen zu lassen, mag als Willkür erscheinen, die Quellen suggerieren aber diesen überdies sinnfälligen Einstieg.

\section{Die hiesigen Lassalleaner}

Um den Dänen derart als „Arbeiter” von Kaub, Hess und Schily zu unterscheiden, bedarf es kaum skrupulöser Definition: von jenen war wohl keiner in den sechziger Jahren gezwungen, sich Aufenthalt, Fleiss und Wohlverhalten regelmässig testieren zu lassen. Petersens livret d'ouvrier ${ }^{2}$ zeigt ihn seit Ende 1859 ziemlich ständig in Paris. Auch wenn diese Quelle keineswegs von überwältigender Zuverlässigkeit ist ${ }^{3}$ in der zweiten Hälfte des 19. Jahrhunderts bestimmt sie ihren dann mindestens 45-jährigen Besitzer wohl hinreichend als Lohnarbeiter.

Seine betont subjektiven Impressionen und Reflexionen von Anfang 1864 können die Ausgangssituation andeuten. Nach einem Aufenthalt

1 Vgl. etwa Schilys Ernennung zum Bevollmächtigten des Generalrats in Paris im März 1865: Minutes 1864-1866, S. 79 und 387-389; MEW, Bd 16, S. 82-83 und Noten.

2 Ausgestellt: Paris 22.VII.1856 (Nachlass Motteler 3038).

3 Z.B. bescheinigt die Firma Franck-Alexander in Paris am 12.V.1873, sie habe Petersen „,depuis l'an 1863, jusqu'à ce jour" beschäftigt; dessen häufige Reisen in der Zwischenzeit haben hier keine Spur hinterlassen. 
in der Schweiz, wo er die Aktivitäten der deutschen Vereine aufmerksam, aber nicht ohne spöttische Distanz, beobachtet hat, ${ }^{1}$ resumiert er die Erfahrungen von fünf Monaten Paris:

„Die kleine Welt Paris ist jedoch so arm an politisches Leben, dass man eigentlich nicht hier lebt sondern nur vegetirt. Alle die grosse schöne deutsche hiesigen Gesang Vereine leiden Mangel daran. [...] Man ist hier so gewöhnt hinter jedem Bierglas ein Mouchard zu erblicken, dass erst einige Gläser ausgeleert muss werden, bis sich die Zunge in freies Lauf setzt. In jedem Café und überall trinkt man Bokbier, und doch erfolgt darnach keine kräftige Stösse - höchstens bittere Mine."

Polizeilich kontrolliert und vorwiegend apolitisch ist dies deutsche Vereinsleben doch der Rahmen, in dem Petersen und einige Freunde - unter ihnen wohl auch Schily - zunächst ohne sichtbare Erfolge eine ungeduldige Agitation betreiben:

„Wir haben allerlei Versuche angestellt die hiesigen Deutschen zu Dies und Jenes $\mathrm{zu}$ bringen. Ich glaube aber, dass wir selber $\mathrm{zu}$ viel Durst und zu wenig Ausdauer dafür haben."

Auffällig, wenn man den Zeitpunkt - „Bismarck steht schlagfertig an der Eider" - und Petersens Nationalität bedenkt, wie stark die Hoffnung bei aller Skepsis darauf gerichtet bleibt, ein Umschwung in Deutschland könne vielleicht doch noch Bewegung in das ,europäische Marionettentheater" bringen:

„Ich in dem guten Glauben an die deutsche Zukunft, glaube am Ende doch dass selber der gemütliche Sauerkraut fressende deutsche Bourgeois am Ende doch die Galle überlaufen kann wenn das Mass zu voll wird - aber wann wird es zu voll?"

Vielleicht ist es nur konsequent, dass die Lassalle'sche Agitation und der ADAV in diesem Bild noch keinen Platz finden. ${ }^{2}$

Im Spätsommer 1865 war dann allerdings die Parteinahme für den ADAV als „Lassalleanische Partei" mit deutlicher Spitze gegen

1 Petersen an Becker, Freiburg (Schweiz) 29.VII.1863 (D II 591) über die dortige Fahnenweihe: „Die Fahne des deutschen Vereins ist sehr schön, nicht allein, weil sie schön gemalen ist und $180 \mathrm{f}$ kostet, sondern weil sie die schöne Devise, ,Freiheit, Gleichheit, Brüderlichkeit' trägt."

2 Petersen an Becker, Paris 27.I.1864 (D II 592); bei Zitaten aus Petersens in Handschrift, Stil und Orthographie gleichermassen charakteristischen Briefen wurde abweichend vom sonstigen Verfahren in Fällen zweifelhafter Lesung die orthographisch korrekte Form gewählt, eindeutig lesbare Abweichungen wurden jedoch beibehalten. 
Marx, Joh. Ph. Becker und deren Stellungnahme in den Fraktionsauseinandersetzungen nach Lassalle's Tode vollzogen; ${ }^{1}$ der Briefwechsel mit dem alten Freunde in Genf schlief für einige Jahre ein. ${ }^{2}$ Mittelbar bestand der Kontakt jedoch weiter, wenn man es als Kontakt werten will, dass „Petersen u. 3 Konsorten” 1866 immerhin die Hälfte der ersten acht Pariser Abonnenten des Vorbote stellten, ${ }^{3}$ diesen auch regelmässig weiter über Schily bezogen und - abrechneten. ${ }^{4}$

Man wird einer Gruppe, die hauptsächlich aus Arbeitern besteht, sich regelmässig trifft, gewisse sozialistische Überzeugungen teilt und bei bestimmten Gelegenheiten gemeinsam in Aktion kommt, für die sechziger Jahre des vorigen Jahrhunderts den Charakter der selbständigen proletarischen Organisation kaum absprechen können. Auch dann nicht, wenn - wie es später einer ihrer Wortführer formuliert die französischen Vereinsgesetze „uns Sozialisten [hindern] unsere Gesinnungen hier öffentlich an den Tag zu legen”. S Seit Anfang 1867 spätestens wird eine solche Gruppe erkennbar: „Für Paris” annoncieren seit der Neujahrsnummer im Social-Demokrat die ,hiesigen Lassalleaner" in unregelmässigen Abständen, meist mindestens einmal monatlich, ihr wöchentliches Treffen, zunächst im Café Buck in der rue de la Verrerie, dann seit April für einige Jahre in der Brasserie Felsz, 37, rue du Marché St. Jean. ${ }^{6}$ Annähernde Grösse und zahlenmässige Entwicklung dieser Gruppe lassen sich bisher nur an einer Reihe indirekter Hinweise ablesen. Als im Mai 1867 ein preussisch-französischer Krieg um Luxemburg drohte, Hess und Petersen in einem der deutschen Arbeitervereine in Paris eine Protestadresse veranlassen wollten, letzterer aber in Zweifel war, ob „,der liebe deutsche Michel sich stark daran beteiligt", erwog er offensichtlich, den Zögernden dadurch nachzuhelfen, dass einige seiner Freunde unterzeichneten, ehe mit der Sammlung weiterer Unterschriften begonnen wurde. Auf „ein Dutzend Namen von Lassalleanern" konnte er wohl ohne nähere Rücksprache rechnen.7 Seine Mitteilung an Hess zum Jahreswechsel 1868-69, der Social-Demokrat habe "mit diesem Quartal [...] 70 Abonnenten in Paris", ${ }^{8}$ war vielleicht eine Übertreibung; Schily übertrieb ein halbes

1 Petersen an Hess, Paris 16.IX [1865], in : Moses Hess, Briefwechsel, hrsg. v. E. Silberner..., Den Haag 1959 [weiter zitiert als: HBW], S. 503.

2 Wenigstens finden sich in Beckers Nachlass keine Briefe von Petersen aus der Zeit zwischen Januar 1864 und Mai 1868.

3 Schily an Becker, Paris 8.VI.1866 (D II 966).

4 Vgl. z. B. Schily an Becker, 27.V.1868 und 1.VII.1869 (D II 980 und 981).

5 Leo Frankel in seinem Bericht über die Pariser Lassallefeier von 1868, in: Social-Demokrat [weiter zitiert als: Soc.-Dem.], Jg. IV, $\mathrm{Nr} 110$ (20.IX.1868).

6 Vgl. Soc.-Dem., Jg. III, Nr 1 (1.I.1867) und Nr 49 (21.IV.1867).

7 Petersen an Hess, Paris 4.V.1867, HBW, S. 547-549.

8 Petersen an Hess, Paris 1.I.1869, HBW, S. 573-574. 
Jahr später sicherlich nicht, wenn er meinte: „Der Social-Demokrat grassiert zu stark unter den hiesigen deutschen Arbeitern, hat circa 3040 Abonnenten". 1

Im Jahrgang 1868 des Social-Demokrat wird dank Petersens regelmässigen Zuschriften exemplarisch deutlich, wie man - das Vorbild deutscher Auslandsvereine mit dem lassalleanischen Organisationsstil kombinierend - ein regelrechtes „Vereinsleben” entwickelte. Dies kennt, neben den montäglichen Versammlungen bei Felsz, zwei deutliche Höhepunkte. Bei einer „Abendunterhaltung” im März bringt man nach wochenlangen Vorbereitungen ${ }^{2}$ - neben ",heitern und ernsten Vorträgen und Deklamationen [...] Gesang und Musik" - Schweitzers Agitationsstück Ein Schlingel zur Aufführung. ${ }^{3}$ Ein sonntäglicher Ausflug in den Bois de Vincennes mit anschliessender Gedenkfeier ist der „Feier des Todestages von Ferdinand Lassalle” gewidmet.4 Verständlich, dass gerade bei dieser Gelegenheit die lassalleanischen Aspekte des Sozialismus der Pariser Gruppe besonders deutlich werden: wenn Petersen Lassalle als den feiert, dessen „Worte das Klassenbewusstsein der deutschen Arbeiter erweckten", Czilinsky eine Ansprache hält, der „,die Worte Lassalle's: ,Ihr Arbeiter seid der Fels, auf welchem die Kirche der Gegenwart gebaut werden soll' zu Grunde lagen", Staade schliesslich das Verhältnis zwischen Kapitalherrschaft und "Lohnschreiberpresse" behandelt, werden unverkennbar Motive der Lassalle'schen Agitation aufgenommen. Leo Frankel - inzwischen als Korrespondent des Social-Demokrat gewonnen - knüpft an aktuelle Diskussionen der Pariser Arbeiter an und spricht über die Frauenfrage. Die alt-proudhonistisch ablehnende Haltung zur Frauenarbeit, die er dort beobachtet, ${ }^{5}$ untermauert er mit der Lassalle'schen Forderung auf den vollen Arbeitsertrag - werde dieser gewährt, werde jene überflüssig.

Mit der Politik der deutschen Organisation verbindet die Pariser Lassalleaner ein selbstverständliches Solidaritätsverhältnis. Die Anwesenheit eines Delegierten des ADAV in Paris ist schon im August 1867 natürlicher Anlass dazu, eine zusätzliche Versammlung abzu-

1 Schily an Becker, Paris 1.VII.1869 (D II 981).

2 Die erste Ankündigung findet sich in einer Notiz aus Paris vom 14.I.1868, in: Soc.-Dem., Jg. IV, Nr 10 (22.I.1868).

3 Vgl. Petersens Bericht vom 3.III.1868 in: Soc.-Dem., Jg. IV, Nr 32 (13.III. 1868).

4 Am 13.IX.1868; vgl. die Anzeige in: Soc.-Dem., Jg. IV, Nr 106 (11.IX.1868), und Frankels Bericht, ebd., Nr 110 (20.IX.1868). In folgenden Jahr führte man die Feier am 19.IX. durch; vgl. den Bericht von Petersen in: Soc.-Dem., Jg. V., Nr 113 (26.IX.1869).

5 Vgl. seine Korrespondenz ,f. Paris, 14. September (Versammlungen zur Besprechung der Frauenarbeit)", in: Soc.-Dem., Jg. IV, Nr 110 (20.IX.1868). 
halten.1 Petersens Korrespondenzen im Social-Demokrat des Jahres 1868 sind oft weniger Bericht aus Paris als Grussadresse und Solidaritätserklärung aus in der Politik des Vereins in Deutschland gegebenem Anlass;" „Ein Wort zur Generalversammlung” in Hamburg unterschreiben am 18. August für ,die hiesigen Parteigenossen" die schon genannten Redner der Lassalle-Feier des gleichen Jahres und $\mathbf{K}$. Goetz; $;^{3}$ gleiches tut dann wieder Petersen zum Allgemeinen Deutschen Arbeiterkongress im September. ${ }^{4}$ Darauf, dass es sich um mehr als verbale Solidarisierung handelte, weisen die relativ zahlreichen Abonnenten des Social-Demokrat, die regelmässig wiederholten - bezahlten Anzeigen und Sammlungen, die man zu gunsten in Deutschland verfolgter Lassalleanischer Agitatoren veranstaltete, ${ }^{\mathbf{5}}$ hin. Man betrachtete diese finanziellen Beiträge sicher nicht als den unwichtigsten Teil der eigenen Aktivität: nicht beiläufig schliesst Petersens Bericht über jene „Abendunterhaltung” nicht nur mit „Brudergruss und Handschlag", sondern auch mit der „Zusicherung, dass wir immer die alten Beisteurer bleiben, die nicht zurückstehen wollen". ${ }^{6}$

Der bisherige Eindruck, die Aktivitäten der Gruppe um Petersen seien hauptsächlich nach innen einerseits, nach Deutschland andererseits gerichtet gewesen, ist allerdings zu korrigieren. Die Richtung der Korrektur war schon in dem gemeinsamen Vorgehen von Petersen und Hess während der Luxemburg-Krise angedeutet. Allem Anschein nach boten verschiedene deutsche Vereine in Paris - ihrem Ursprung nach eher gesellig, höchstens einer allgemein demokratischen Tradition verbunden - in den späten sechziger Jahren den Lassalleanern, und nicht nur ihnen, zunehmend die Möglichkeit zu Diskussion und Agitation in weiterem Rahmen. Ganz deutlich wird das zum Jahrwechsel 1868-69: im Turnverein und in der Germania, einem Gesang- und Arbeiterverein, werden "soziale Fragen" diskutiert; die Verabredung Petersens mit Schily und Hess ${ }^{7}$ wird sich nicht nur auf gemeinsame Teilnahme bezogen haben - später jedenfalls bereitete man offensichtlich auch Diskussionsbeiträge und deren Unterstützung durch Teilnahme der "Mehrzahl der hiesigen Lassalleaner" vor. ${ }^{8}$ Die Frage, ob sich damals in den Vereinen ein sozialistisch gerichteter Politisie-

1 Vgl. Petersen an Hess, Paris 16.VIII.1867, HBW, S. 554-555.

2 So z.B. sein "Neujahrsgruss", in : Soc.-Dem., Jg. IV, Nr 4 (8.I.1868), und „Zum Nothstand", ebd., Nr 14 (31.I.1868).

3 Ebd., Nr.99 (23.VIII.1868).

- Ebd., Nr 107 (13.IX.1868).

- Ebd., Nr 14 (31.I.1868).

- Ebd., Nr 32 (13.III.1868).

- Petersen an Hess, Paris 1.I.1869, HBW, S. 573-574.

8 Vgl. Petersen an Hess, Paris 19.XI.1869, HBW, S. 588-589. 
rungsprozess abspielte und welche Rolle die Pariser Lassalleaner dabei spielten, kann hier vorläufig nur gestellt werden.

Augenfälligstes Ergebnis dieser Arbeit in den deutschen Vereinen war es jedenfalls, dass Petersen im Sommer 1868 Leo Frankel, der in der Germania einen Vortrag über die „Vortheile der Arbeitseinstellungen" gehalten hatte, ${ }^{1}$ zur regelmässigen Mitarbeit am Social-Demokrat brachte. Mit ihm, der nach eigener Aussage früher Sozialist als Lassalleaner gewesen war, ${ }^{2}$ gewann die Pariser Gruppe einen schreibgewandten Wortführer, der Social-Demokrat einen Pariser Korrespondenten, der die Entwicklung der französischen Arbeiterbewegung sehr aufmerksam und gut informiert verfolgte. Interessante Fragen, wie die nach dem Informationswert von Frankels an Lassalle'schen Theoremen orientierter Berichterstattung über den Übergang der französischen Arbeiterbewegung vom älteren Proudhonismus zum revolutionären Kollektivismus, oder danach wie die Pariser Lassalleaner durch diese Entwicklung ihrerseits beeinflusst wurden, können hier nur angedeutet werden.

Immerhin gibt es einige Hinweise, die auch ohne intensivere Interpretation der den Pariser Berichten von Petersen und Frankel zugrunde liegenden theoretischen Position, die Vermutung rechtfertigen, dass sich im Jahre 1868 eine Annäherung zwischen den Pariser Lassalleanern und der IAA angebahnt habe.

Die Solidaritätsaktionen zu Gunsten der streikenden Genfer Bauarbeiter im Frühjahr 1868 führten im Mai zu direktem Kontakt mit einer Gruppe aus der Schweiz zugewanderter Internationalisten. Unabhängig davon, aber ungefähr gleichzeitig nahm auch Petersen seine Korrespondenz mit Joh. Ph. Becker wieder auf und meinte, er ,habe nie ein[en] wesentlichen Unterschied zwischen diesen beiden Richtungen finden können, vom prinzipiellen Standpunkte aus". 3 Die schon erwähnte Adresse an die Hamburger Generalversammlung definiert das Verhältnis beider im Verständnis der Pariser näher: der ADAV als „das Banner [...] um dass sich der Arbeiterstand in Deutschland scharen wird", um dann, „wenn das jetzige Elend der Arbeiter einst weichen wird den Forderungen der Zeit”, ,ein kräftiges Glied [zu] sein in der Kette der allgemeinen Verbrüderung der europäischen Arbei-

1 Vgl. Soc.-Dem., Jg. IV, Nr 78 (5.VII.1868).

2 Vgl. Frankel an Becker, Paris 22.II.1870 (D II 629); dort auch Frankels Bericht über seine ersten Kontakte mit den Pariser Lassalleanern.

3 Petersen an Becker, Kopenhagen s.d. (D II 623); nach einer Nachricht im „Briefkasten" von Soc.-Dem., Jg. IV, Nr 51 (29.IV.1868) befand sich P. zu diesem Zeitpunkt in Dänemark, die ,Nachträgliche Bescheinigung”, ebd., Nr 77 (3.VII.1868) zeigt ihn Anfang Juli wieder in Paris. 
ter".1 Während der Schweizer Frühjahrsstreiks des folgenden Jahres zeigen die im Vorbote quittierten Beträge - insgesammt 308,50 Franken, ${ }^{2}$ dass man sich zur IAA nicht nur als zur Verbrüderung der Zukunft, sondern auch $\mathrm{zu}$ ihr als aktueller internationaler Kampforganisation verhielt. Den Beitrag wollte Petersen verstanden wissen „als ein geringer Beweis für unser Teilnehmen, als Glied der grossen Brüderfamilie, für unser allgemeines Interesse". ${ }^{3}$ Wiederum ein halbes Jahr später, als der damalige Sekretär des Generalrats der Assoziation, Georg Eccarius, auf der Rückreise vom Basler Kongress nach Paris kam, hatte mindestens Leo Frankel anscheinend die Absicht, die Verbindung seiner Freunde mit der IAA auch organisatorisch zu vollziehen. ${ }^{4}$ Die „100 Karten der Internationalen”, die Frankel von Eccarius erhielt, blieben aber für nahezu ein halbes Jahr ungebraucht; vorteilhaftes Ergebnis dieser Verzögerung war, dass die dann gefundene Form der "deutschen Sektion" nicht nur Pariser Lassalleaner zum Anschluss an die Assoziation brachte, sondern zugleich andere Organisationsansätze deutscher Arbeiter in Paris einschloss.

\section{Internationale Mitglieder}

Als der aus der Rheinpfalz gebürtige Schneider Philipp Peter im Oktober 1867 nach Paris übersiedelte, war er für die Internationale sicher kein unbeschriebenes Blatt mehr. Im Juli 1866 als Mitgründer, dann als erster Präsident der deutschen Sektion in La Chaux-deFonds ${ }^{5}$ erscheint er in seinen Briefen an Becker als einer jener beharrlichen, stets zuverlässigen, gelegentlich überkorrekten Arbeiterorganisatoren, ohne die die Geschichte der IAA nicht denkbar erscheint, die aber in den Personenregistern nur dann erscheinen, wenn sie sich in eine der „,bedeutsameren" fraktionellen Streitfragen verwickeln liessen. So Philipp Peter, der seine Sektion 1866 auf dem Kongress in Genf vertrat ${ }^{6}$ und dort ausgerechnet zur religiösen Frage ausführlicher Stellung nahm; jener Frage also, die Marx für so wenig bedeutsam gehalten hatte, dass er der englischen Delegation riet, die Initiative den

1 Soc.-Dem., Jg. IV, Nr 99 (23.VIII.1868).

2 Der Vorbote, Jg. IV, Nr 2 (Febr. 1869), S. 31 und 32; ebd., Nr 4 (April 1869), S. 58 und 63.

s Petersen an Becker, Paris 30.III.1869 (D II 593).

4 Frankel an Becker, Paris 24.I.1870 (D I 628).

- Vgl. Peter an Becker, La Chaux-de-Fonds 1. und 27.VIII.1866 (D II 560-561); über die Sektionen in La Chaux-de-Fonds vgl. E. Gruner, Die Arbeiter in der Schweiz im 19. Jahrhundert, S. 559 f.

- La Première Internationale, Recueil de documents publié sous la direction de Jacques Freymond, Bd I, Genf 1962, S. 28.

7 A.a.O., S. 53 und 77. 
Franzosen $\mathrm{zu}$ überlassen. ${ }^{1}$ Peter vertrat anscheinend ${ }^{2}$ recht nachdrücklich den Standpunkt der Beckerschen Denkschrift, ${ }^{3}$ wie er auch anderwärts die Haltung der "deutschen" Delegation teilte. ${ }^{4}$

Peters Pariser Briefe aus den Jahren 1867-69 sind wie immer knapp gehalten. Sie geben aber hinreichend Aufschluss darüber, wie er seine zwei Monate nach der Übersiedelung mitgeteilte Absicht, dass er Mitglied der Assoziation bleibe und sich ,auch [...] in Paris weiter um unsere Sache[,] Arbeiter[-]Sache kümmere", 5 verwirklichte. Als deutliche Höhepunkte dieser Tätigkeit treten die Bemühungen zur Unterstützung der Streikbewegungen von Genf (Frühjahr 1868) und Basel (Winter 1868-69) hervor.

Genugtuung ist unverkennbar, wenn Peter und zwei Freunde im Mai 1868 als Ergebnis ihrer Aktivität die nicht unbeträchtliche Summe von 64,10 Franken zu diesem Zwecke absenden können. ${ }^{6}$ Dass sie zunächst unter ihren „Bekannten, Profession Schneider eine kleine Sammlung" veranstalten, wie deren Ertrag, 46,50 Franken, suggeriert anscheinend nicht nur für den heutigen Leser ein vorwiegend syndikales Interesse dieses Freundeskreises: Becker quittierte den Betrag, ohne dass im Brief von einer solchen Organisation die Rede wäre, für den „Schneiderverein Paris”.?

Einen Schritt über diesen Rahmen hinaus bedeutete wohl die Teilnahme der erklärten „Internationalen” an einer Versammlung der Pariser Lassalleaner. Wichtiger als die dort gesammelten 14 Franken erscheint das, was Peter über Verlauf und Ergebnis des Besuches andeutet: Wenn man dort „die Sache der Greve, sowie der Assoziation" diskutierte, so waren beide Fragen im Kreise der Gastgeber zumindest noch umstritten. ${ }^{8}$ Der von jener Seite geäusserte Wunsch,

1 Vgl. MEW, Bd 16, S. 199.

2 Der Bericht des Vorbote schreibt offensichtlich dieselbe Intervention dem Lausanner Delegierten Schlaifer zu; vgl. Vorbote, Jg. I, Nr 11 (Nov. 1866), S. 169. 3 "Denkschrift der deutschen Abtheilung (Genf) der Internationalen Arbeiterassociation [...] Elfter Punkt", ebd., Nr 8 (Aug. 1866), S. 122.

4 So z. B. zu den Fragen „Beschränkung der Arbeitszeit" und ,Stehende Heere” im Kongressbericht des Vorbote, Jg. I, Nr 10 (Okt. 1866), S. 149, und Nr 11 (Nov. 1866), S. 169. Peter gehörte auch zu den Unterzeichnern der Beckerschen Kompromissresolution in der Polenfrage, vgl. den Resolutionsentwurf im Nachlass Jung, $\mathrm{Nr} 44$.

5 Peter an Becker, Paris 26.XII.1867 (D II 568).

- Peter an Becker, Paris 7.V.1868 (D II 574).

7 Der Vorbote, Jg. III, Nr 5 (Mai 1868), S. 68.

8 Erst die VII. Generalversammlung des ADAV vom 23.-26.VIII.1868 revidierte die bisherige Haltung des Vereins in der Streik- und Gewerkschaftsfrage. Da Petersen sich zu diesem Zeitpunkt gerade in Dänemark aufhielt, Frankel seine Korrespondenzen aber erst im Juli aufnahm, fehlt leider ein entsprechender Bericht im Social-Demokrat. 
„eine nähere Verbindung zwischen der internationalen und der deutschen Bewegung" zustande zu bringen, mag als Hinweis auf jene „Durchlässigkeit der Fronten” gelten, die bei den Organisationsversuchen der deutschen Arbeiter in Paris häufiger zu beobachten ist. Wenn Peter in diesem Zusammenhang zwölf der Becker'schen Streikbroschüren ${ }^{1}$ bestellte, so betrachtete er die begonnene Diskussion wohl kaum als abgeschlossen. Ob dieser erste Anlauf direkte Konsequenzen hatte, bleibt offen; Peter abonnierte weiter regelmässig Exemplare des Vorbote, setzte seine Agitation offensichtlich fort und beurteilte das Ergebnis nicht ungünstig: ,im allgemeinen geht alles so ziemlich gut von statten". 2

Greifbares Ergebnis erneuten Kontaktes mit den Lassalleanern und Ausdruck langsamen, aber gleichmässigen Fortschritts ist knapp acht Monate später die für die Streikenden in Basel gesammelte Unterstützung: 88,50 Franken. Einer der beiden Mitunterzeichner vom Mai des Vorjahres hat inzwischen entweder Paris verlassen, oder seine Tätigkeit eingestellt, die Leistungsfähigkeit der „Gruppe Peter” aber ist gewachsen, ihr Einflussbereich hat sich jedenfalls erweitert. Rahmen für diesen Einfluss, zudem Berührungspunkt mit parallelen Agitationsund Organisationsversuchen sind wieder die Versammlungen des deutschen Turnvereins, ,wo jedesmal viel über die Arbeiterbewegung gesprochen wird". Unter den Teilnehmern der letzten Versammlung erwähnt Peter Simon, Hess und Schily, hatte letzteren also wohl spätestens zu diesem Zeitpunkt kennengelernt. ${ }^{3}$

Vielleicht nur Zufall, vielleicht aber auch terminologische Verschiebung, die Änderung von Zielvorstellungen und Erfolgserwartungen ausdrückt, jedenfalls aber mitteilenswert ist die gegenüber dem Vorjahr auffällig veränderte Wortwahl der Begründung der eigenen Solidarität mit dem Baseler Streik:

1868 dankte man den Genfer Genossen für ihre „Ausdauer und Opferwilligkeit”, mit der sie „aller Welt gezeigt” hätten, ,zu welchem Ziele man durch Einigkeit gelangen kann". Als Folge dieses exemplarischen Verhaltens erwartete man ",noch weitere Fortschritte" für "dieAssoziation".

1 Joh. Ph. Becker, Die Internationale Arbeiter-Association und die Arbeitseinstellung in Genf im Frühjahr 1868, Genf 1868.

2 Peter an Becker, Paris 30.V.1868 (D II 576).

3 Peter an Becker, Paris 21.I.1869 (D II 576); Peter erwähnt die Zusammenarbeit mit den Lassalleanern hier nicht; den gleichen Betrag nennt aber Petersen als Ergebnis einer Sammlung ,in Verbindung mit einigen Mitgliedern der deutschen Sektion", in: Soc.-Dem., Jg. V, Nr 7 (15.I.1869); Vorbote, Jg. IV, Nr 2 (Febr. 1869) quittiert: „Fr. 88.50 deutscher Arbeiterverein Paris (Versender Petersen)".

4 Peter an Becker, Paris 7.V.1868 (D II 574). 
1869 in Basel geht es schlicht und einfach um den „Sieg gegen die Bourgeoisie und [das] Kapital”, den "unsere Mitgenossen” "davon tragen werden, oder schon davon getragen haben". ${ }^{1}$

Bis zur Gründung der Sektion liegen keine weiteren Briefe von Peter vor. Dass aber Peter auf Grund seiner bisherigen Tätigkeit in Paris im Sommer 1869 zum organisatorischen Kristallisationskern geworden war, zeigt eine Äusserung Schilys aus dieser Zeit. Sie gibt ausserdem ein anschauliches Bild der bisherigen Ergebnisse, wie der Beschränkungen und Gefahren, denen Versuche, die deutschen Arbeiter in Paris innerhalb der Internationale zu organisieren, unterlagen:

„Hier, d.h. unter den deutschen Arbeitern eine internat. Sekt. zu gründen, können wir den Leuten nicht wohl zumuten; man würde ihnen vielleicht nicht die Ehre einer gerichtlichen Untersuchung u. Verurteilung angedeihen lassen, wohl aber sie per Schub über die Grenze bringen. Indessen könnten Peter u. Genossen so was, wie internationalen Unterstützungsverein für Deutsche, Schweizer, etc. bilden, an welchen sich Zureisende zur Auffindung von Arbeit usw. wenden könnten; und woraus man seiner Zeit, wie auch jetzt schon unter der Hand, was Andres machen könnte; aus der Schweiz herüberkommende internat. Mitglieder müssten in diesem Sinne mit Peter in Verbindung treten. Dann liesse sich auch der Vorbote besser propagieren und würde ich aus meinem Vorrat vollständige Jahrgänge zur Verfügung [...] stellen, wie ich dies bei Petersen getan, jedoch ohne Erfolg; in seinen Kreisen schwört man auf Schweitzer und den Sozialdemokrat."2

Wenn der von Schily vorgeschlagene Weg halbkonspirativer Vorsicht eingeschlagen wurde, so blieb er ein Zwischenspiel, dessen Spuren, wenn vorhanden, nicht erkennbar sind. Dass Philipp Peter ein reichliches halbes Jahr später wie selbstverständlich zu den Gründern der deutschen Sektion zählt, kann nach dem bisherigen kaum noch verwundern.

\section{Bäcker deutsche}

Relativ auf sich gestellt, aber nicht ohne Berührung mit den anderen Organisationsversuchen scheint Joseph Gebert seine Aktivitäten entwickelt zu haben. Eine späte Charakteristik Schilys, der auf Grund der Zusagen des Bäckergesellen einen schnellen Vertrieb der Beckerschen Druckerzeugnisse erhofft hatte und sich darin getäuscht sah, erscheint wenig schmeichelhaft:

2 Schily an Becker, Paris 1.VII.1869 (D II 981). 
„Was eigentlich hinter diesem Gesellen und seinem Agitieren steckt, weiss ich nicht. Periodisch poltert er ungeheuer demokratisch-sozialistisch, gibt u. nimmt Rendez-vous, um grosse Dinge $z u$ vollführen und verschwunden ist $u$. bleibt er dann für lange Zeit; sein letztes von ihm nicht gehaltenes Rendez-vous mag von einem Jahr her datieren."1

Wahrscheinlich hat jene episodische Agitation keine anderen Spuren hinterlassen, ${ }^{2}$ als drei etwas verworrene Briefe an Becker, den Gebert spätestens Anfang 1867 kennengelernt hatte, ${ }^{3}$ dessen Zeitschrift er abonnierte ${ }^{4}$ und dem er berichtete: ,je fais mon possible de propager cette doctrine de fraternité qui prend racine sur notre globe et que rien ne peut arrêter tot [sa?] marche de progrès". ${ }^{5}$

Gebert dennoch in Erinnerung zu bringen, lohnt sich aus zwei Gründen. Wenn er im März 1867 über die Fortschritte der „societé de Secours mutuels" berichtet, macht er die deutliche Ausnahme: nur

„die unverständigen Deutschländer, wo sich in Paris befinden wollen nicht anteilnehmen, denn man berechnet mehr als 400 Bäcker deutsche", 6

im Sommer 1869 ist seine Aktivität darauf gerichtet „de former une section entre les ouvriers boulangers de Paris"; beides kann vorläufig als Hinweis auf eine Berufsgruppe mit einem relativ hohen Anteil deutscher Arbeiter gelten. Zudem gibt der Brief vom 12.VIII.1869 zusätzlichen Aufschluss über die „hiesigen Lassalleaner”; Gebert, der sich offensichtlich inzwischen zu dieser Gruppe rechnet, berichtet:

„La section Petersé se réunit régulièrement deux fois par semaine, une fois rue Keller7 et l'autre rue Valois ${ }^{8}$."

Gebert erwartet, mit einem Grussschreiben Beckers anlässlich eines Ausflugs nach Argenteuil neue Abonnenten für den dem Schweitzerschen ADAV doch gewiss nicht freundlichen Vorbote zu gewinnen; Anzeichen dafür, dass der Prozess der Loslösung der Pariser Lassalleaner

1 Schily an Becker, Paris 21.V.1870 (D II 984).

2 Gebert erscheint z.B. nicht im DBMOF.

$3 \mathrm{Vgl}$. Gebert an Becker, Paris 18.III.1867 (D I 794).

4 Quittungen in Vorbote, Jg. III, Nr 9 (Sept. 1868), S. 144, und Jg. IV, Nr 1 (Jan. 1869), S. 16.

5 Gebert an Becker, Paris 12.VII.1869 (D I 795).

6 Gebert an Becker, Paris 18.III.1867 (D I 794).

7 Über diese zweite wöchentliche Versammlung liess sich bisher nichts feststellen.

${ }^{8}$ Im Café Charles, 40, rue de Valois; vgl. die Annonce in Soc.-Dem., Jg. V, Nr 77 (4.VII.1869). 
von der Berliner Führung schon begonnen hat? Die Selbstverständlichkeit schliesslich, mit der Gebert über die „section Petersé” berichtet, lässt erkennen, dass die regelmässigen Zusammenkünfte der Pariser Lassalleaner ungeachtet aller gesetzlichen Beschränkungen im Bewusstsein ihrer Teilnehmer den Charakter eines organisatorischen Zusammenschlusses gewonnen hatten, der mit dem in der Internationale gebräuchlichen Begriff bezeichnet wurde. Diese organisatorische Identität wird den Pariser Lassalleanern wenige Monate später auch von aussen bestätigt: wenn Varlin in kritischer finanzieller Situation sich um Streikunterstützung auch durch den ADAV bemüht, sucht er ebenfalls selbstverständlich die Vermittlung der "section des démocrates socialistes allemands de Paris".1

Unter den verschiedenen Ansätzen selbständiger proletarischer Organisation unter den Deutschen in Paris hatte gegen Ende der sechziger Jahre derjenige der Lassalleaner einen Vorsprung erzielt, der diese Gruppe einerseits zum Gravitationspunkt für gleichgerichtete Versuche machte, andererseits aber die organisatorische Ratifikation der Beckerschen Aussage vom Oktober 1868, in Paris hätten sich „die sogen. Lassalleaner mit den aus der Schweiz dorthin gewanderten Internationalen zu gemeinsamer Bestrebung vereinigt", um nahezu eineinhalb Jahre verzögerte. ${ }^{2}$ Schilys biblisch-plastische Vision einer Lösung des deutschen Fraktionsstreites

„Um diesem verbissenen Lassalleanismus beizukommen, müsste man dessen jetzigen Krakehl mit dem Liebknechtentum in Deutschland für die Internationale auszunutzen suchen, indem man diese als die Arche des Bundes oder Noahs darstellt, in welche die Bekehrten aus beide Lagern mit Sack u. Pack friedlich einziehen können, die Lassalleaner mit all ihren Lassall'schen Bibeln und Fibeln, Testament und sonstigen Reliquien des ,grossen Toten', unter welchen Reliquien auch die Gräfin Hatzfeld mit den Kassen zu begreifen wäre, so wie die Liebknechte ebenfalls mit ihren ganzen Inventarium, incle Welfische Kassen, die zur praktischen Ausbildung des Strike-Systems so viel wie möglich zu verwenden wäre."3

erfuhr eine ausserdeutsche Teilverwirklichung gerade dort, wo er sie

1 Varlin an Aubry, Paris 4.XI.1869, in: Troisième procès de l'Association Internationale des Travailleurs à Paris, Paris 1870 [weiter zitiert als: III. Procès], S. 27.

Vorbote, Jg. III, Nr 10 (Okt. 1868), S. 159.

s Schily an Becker, Paris 1.VII.1869 (D II 981), also kurz nach Schweitzers „Staatsstreich" vom 18.VI.1869. 
für unmöglich gehalten hatte, ${ }^{1}$ erst dann aber, als mindestens der Kern der Pariser Lassalleaner aufgehört hatte, sich mehr oder weniger vorbehaltlos als Teil des ADAV zu betrachten und dessen Führung in allen nationalen und internationalen Streitigkeiten solidarisch $\mathrm{zu}$ unterstützen.

\section{II}

Mit Leo Frankels Briefen an Becker liegt für die Bestimmung dieses Zeitpunktes, wie für die Gründungs- und kurze Lebensgeschichte der Sektion eine ungleich reichhaltigere Quellengruppe vor, als für die Skizze ihrer Vorgeschichte zur Verfügung stand. Die Möglichkeit aber, Frankels Aussagen auf Grund anderer Quellen zu kontrollieren, besteht für diesen Zeitpunkt vorläufig nur an wenigen Stellen. Zwei Tendenzen, die den Briefen aus dem Jahre 1870 gemeinsam sind, sollten darum vermerkt und gegebenenfalls zur Relativierung dieser Aussagen benutzt werden. Einmal ist bei Frankel ein Zug zu - gelegentlich recht weitschweifiger - kaum verdeckter Apologie unverkennbar; in der Korrespondenz des bisherigen Exponenten des Pariser Lassalleanismus mit dem ihm bis dahin sicher nicht nur ,in persönlicher Beziehung" "fernstehenden" Becker ${ }^{2}$ ist dies auch kaum verwunderlich. Zum anderen hat das jedenfalls ungebrochene Selbstbewusstsein Frankels zur Folge, dass die Geschichte der Sektion hinter der Figur ihres „Gründers" und korrespondierenden Sekretärs gelegentlich verblasst.

\section{Mit der Avantgarde der sozial-republikanischen Armee}

Als Frankel sich zur Jahreswende 1869-70 zum ersten Male an Joh. Ph. Becker wendet, tut er dies mit der erklärten Absicht, ,,bald eine internationale Sektion Deutscher hier [zu] gründen", und kann zunächst auf etwa 30 Mitglieder rechnen. ${ }^{3}$ Da die Sektion Frankel später zu ihrem Sekretär ${ }^{4}$ und Delegierten im Pariser Föderalrat ${ }^{5}$ wählte, wird man ihm die Rolle des Initiators kaum absprechen können. Schon in diesem ersten Brief sind aber auch die Voraussetzungen seiner Initiative hinreichend angedeutet: mindestens der Kern der Pariser Lassal-

1 Siehe oben S. 115.

2 Frankel an Becker, Paris 1.I.1870 (D I 627).

3 Ebd.

4 So unterzeichnet Frankel zuerst seinen Brief an Becker vom 13.III.1870 (D I 630).

5 Vgl. Frankels Verteidigungsrede, bes. die Begründung seiner Aussageverweigerung in der Voruntersuchung, III. Procès, S. 213-215. 
leaner hatte zu diesem Zeitpunkt mit der Berliner Führung gebrochen. Frankel selbst, der durch seine Mitarbeit an Social-Demokrat, Volksstimme und Volkswille $\mathbf{1}^{\mathbf{1}}$ zum Wortführer der Gruppe in der deutschsprachigen Arbeiterpresse geworden war, ${ }^{2}$ hatte - die Generalversammlung des ADAV, vor allem „Neuwahl des Vorstandes namentlich des Präsidenten u. Sekretärs” abwartend - vorläufig seine „Beziehungen zu dem Sozialdemokrat gelöst". 3 Der bisher unbekannte Henri Bachruch, ${ }^{4}$ der in den Konflikten der letzten Monate durch Stellungnahmen in französischen Blättern in eine ähnliche Lage geraten war, ,, ist mit [Frankel] einverstanden". Weniger augenfällig, aber angesichts der organisatorischen Rolle, die Petersen in den vorangegangenen Jahren gespielt hatte, sicherlich ebenso wichtig war es, dass dieser "seine Stelle als Bevollmächtigter abgelegt"5 und damit die Beziehungen zum ADAV auch formell gelöst hatte.

Für Vorgeschichte und Motivation dieses Bruchs steht vorläufig nur Frankels Lesart zur Verfügung. Bemerkenswert dazu ist sein späteres Zeugnis: „An der Sache haltend, habe ich mit Freude die Vereinigung der Lassalleschen Parteien begrüsst”. ${ }^{6}$ Schweitzers „Staatsstreich" vom Juni 1869 war von diesem Standpunkte aus jedenfalls nicht der gegebene Anlass; die Gründung der SDAP in Eisenach konnte kaum auf sympathisierende Reaktion rechnen. Erst neuerliche Konflikte innerhalb der „vereinigten” Lassalleanischen Partei liessen in Frankels Augen wenige Monate später die ganze Operation als Komödie erscheinen und er äussert ex post ,als Beklatscher von Komödienspielern will ich nicht erscheinen".7 An eine Annäherung an die Eisenacher Partei aber war zu diesem Zeitpunkt umso weniger zu denken, als Frankel und mit ihm die Pariser Lassalleaner - als bonapartistische Soutenus verdächtigt - in eine Polemik mit dem Pariser Korrespondenten des Volksstaat verwickelt worden waren, in der Frankel Moses Hess nichts schuldig blieb und alle in den deutschen Fraktionsstreitigkeiten der sechziger Jahre üblichen unerfreulichen Register gezogen wurden. ${ }^{8}$

Durch diesen Konflikt verständlicher werden die Wendungen, mit

1 Vgl. M. Aranyossi, a.a.O., S. 16 f.

2 Vgl. Liebknecht an Hess, 28.XI.1869, HBW, S. 589, und Marx an Engels, 14.IV.1870, MEW, Bd 32, S. 474.

3 Frankel an Becker, Paris 1.I.1870 (D I 627).

4 Zu Bachruch vgl. vorläufig DBMOF, Bd IV, S. 167-168.

5 Frankel an Becker, a.a.O.

- Ebd.

7 Ebd.

8 Vgl. dazu E. Silberner, a.a.O., S.613-615; dort und in HBW, S. 589f. auch Hinweise auf Bachruchs Anteil an dieser Polemik. 
denen Frankel nach dem Bruch mit Schweitzer die eigene Position - und wohl auch die seiner Pariser Freunde - definiert. Der in der Auseinandersetzung mit der gegnerischen Fraktion lassalleanischgebräuchliche Vorwurf ehrgeizigen Literatentums wird nun unverkürzt auch gegen Schweitzer und sein Gefolge gerichtet. Gemeinsamer Nenner, unter dem sich das unklare Verhältnis des Volksstaat zur bürgerlichen Demokratie, wie die antisemitische Demagogie des Social-Demokrat fassen lässt, ist die Beobachtung,

„dass sich die sämtliche Arbeiterpartei Deutschlands von Literaten führen lässt, welche da sie sämtlich persönlich sich gegenseitig rancune tragen, die Arbeiter gegeneinander hetzen anstatt gemeinsam gegen Regierung u. Bourgeoisie aufzutreten".1

Die Konsequenz dieses Urteils, mit dem sich die wichtigste Gruppe organisierter deutscher Arbeiter in Paris, die ihre Aktivität bis dahin vorwiegend an der Entwicklung der Arbeiterbewegung in Deutschland orientierte, abrupt von dieser Bewegung und ihren Fraktionen abwandte, hat Frankel persönlich-drastisch formuliert: sei mit der deutschen Sektion in Paris die neue organisatorische Form für diese Gruppe gefunden, so „möge man sich dann sämtliche Führer in Deutschland einsalzen". 2

Als Abkehr negativ ausgedrückt deutet sich hier eine Haltung an, deren positive Wendung wahrscheinlich für die deutsche Sektion nicht untypisch war, auch wenn sie hier nur in Frankels Worten wiedergegeben werden kann. Beurteilte man die Situation so:

,jetzt gilt es oder niemals energisch vorwärtszuschreiten, jetzt gilt es die Arbeiterarmee aufzustellen u. dann en avant!",

war man in täglicher Berührung mit den Kämpfen und dem Aufschwung der französischen Internationale im letzten Jahre des zweiten Kaiserreiches, war man selbst inzwischen als Delegierter der deutschen Sektion im dritten Prozess gegen die Pariser Internationale verurteilt worden, so lag es wohl nahe, ,in Deutschland bloss Zeitungsartikel an Stelle eines mutigen nicht gefahrscheuenden Auftreten [zu] finden". Der Vergleich schien die Erwartung zu bestätigen, ,dass die Bewegung in Frankreich zum Ausbruch kommen werde". ${ }^{4}$ Vielleicht war der Gedankengang stärker durch psychologische Eingängigkeit für die unmittelbar Beteiligten als durch analytische Schärfe. Seine Pariser Genossen

1 Frankel an Becker, a.a.O.

2 Ebd.

3 Ebd.

4 Frankel an Becker, Paris 14.VII.1870 (D I 635). 
wenigstens hatte Frankel schon im April 1870 mit ihm überzeugen können. Der Aufruf der deutschen Sektion „An die Arbeiter Deutschlands!" (Dokument III) brachte diese Überzeugung deutlich zum Ausdruck. Deutlich genug jedenfalls, dass die Redaktion des Felleisen dem Abdruck einen Kommentar folgen liess, der die Unterstützung des Streiks in Creuzot zwar dringend empfahl, die Ansicht aber, die der französischen Arbeiterbewegung die Rolle der Avantgarde zuschrieb, als „Messiasglaube” kritisierte. Die Begründung zeigt, dass man in Zürich gerade die materiellen Konsequenzen der dort vorgeschlagenen Konzentration aller Kräfte auf einen Punkt fürchtete:

„die Verpflichtung zur Unterstützung der Gefährdeten [kommt] dem internationalen Verbande nur in dem Masse wirklich zu statten, als sie zugleich der nationalen Brüder nicht vergisst [...]. Darum lasst uns den Brüdern in Wien beistehen und die Brüder in Creuzot nicht vergessen!'”

Späteres Zeugnis für die persönliche Option Frankels für die französische Bewegung ist die Begründung seines Entschlusses, während des Krieges in Paris zu bleiben, obwohl er bei seiner Rückkehr im September 1870 die Existenz der deutschen Sektion durch die Ausweisungsdekrete beendet fand (vgl. Dokument X). Die Revolution des 18 . März 1871 erschien Frankel dann als Bestätigung seiner Vorhersage und Rechtfertigung der eigenen Haltung; ausdrücklich bezieht er sich auf jenen Aufruf der Sektion, in dem er

„die Unterstützung der französischen Arbeiter besonders betonte, weil ich in dem hiesigen Proletariat die Avantgarde der sozialrepublikanischen Armee erblickte, eine Meinung die dem ,Felleisen' nicht besonders zu gefallen schien.

Gegenwärtig beginnt meine Meinung in Wahrheit überzugehen...."2

\section{Innere Vereinsangelegenheiten}

Der Organisationsgeschichte der deutschen Sektion ist hier vorgegriffen worden, um neben den organisatorischen auch die ideologischen Voraussetzungen ihrer Gründung und späteren Aktivität zumindest anzudeuten. Die Gründung selbst kam nicht so reibungslos zustande, wie es nach Frankels optimistischen Äusserungen vom Neujahrstag 1870 zu erwarten gewesen wäre. Widerstand leistete, obwohl die aktivsten

1 Felleisen, Organ der deutschen Arbeiterbildungsvereine in der Schweiz, Jg. IX, Nr 17 (23.IV.1870), S. 4.

2 Frankel an Marx, Paris 30.III.1871, in M. Aranyossi, a.a.O., S. $260 f$. 
bisherigen Mitglieder Frankel folgten, mindestens ein Teil der lassalleanischen Gruppe; gerade von dieser Seite musste sich Frankel jetzt den Vorwurf gefallen lassen, dass er ,aus reiner Eitelkeit und Wichtigmacherei (Stil Schweitzers) die Gründung der intern. Sektion durchführen wolle."1 Es kam anscheinend zu einer regelrechten Spaltung, denn auch nach der Gründung der Sektion finden sich im Social-Demokrat Annoncen, nach denen sich in Paris „Die hiesigen Lassalleaner (Mitglieder des Allgemeinen Deutschen Arbeitervereins)" weiterhin regelmässig versammelten. ${ }^{2}$ Hinweis auf diese Auseinandersetzungen ist ein eigentümlicher Widerspruch in den verfügbaren Quellen. Am 22. Februar betrachtet sich Frankel als ,von den hiesigen Parteigenossen beauftragt”, Becker die Gründung der Sektion als „vollbrachte Tatsache, die allerdings nicht ohne Kämpfe erfolgte, und deren Bestehen auch nicht künftighin unangegriffen bleiben wird" mitzuteilen. Noch fünf Tage später, inzwischen hat eine weitere Versammlung stattgefunden, weiss der in Organisationsfragen pedantischere Philipp Peter zwar auch von Fortschritten zu berichten, eine formelle Gründung aber steht in seinen Augen noch aus und kann durch ein rechtzeitiges Schreiben von Becker vielleicht gefördert werden. ${ }^{3}$ Man ist geneigt, der Peterschen Version zu folgen, wenn man beobachtet, dass Frankel erst seinen folgenden Brief vom 13. März auch formell als korrespondierender Sekretär der Sektion unterzeichnet. Was aber in der Versammlung vom 17. Februar, über die Frankel am 22. an Becker berichtete, erreicht worden war, rechtfertigte seine Auffassung der Sachlage mindestens inhaltlich. Wenn 23 „deutsche Sozialisten" sich gemeinsam und als "Mitglieder der internationalen Assoziation” den französischen Protesten gegen die Verhaftung Varlins anschlossen (s. Dokument I), so begriffen schon die Zeitgenossen diesen Schritt als erstes öffentliches Auftreten der Sektion. ${ }^{4}$ Unter den Unterzeichnern finden sich von den bisher Bekannten die Festredner der Lassalle-Feier von 1868 mit Ausnahme von Czilinsky, dazu Bachruch und Philipp Peter. Auffällig ist das Fehlen von Schily, dessen spätere Mitgliedschaft verbürgt ist $^{5}$ und der von einer organisatorischen Regelung, die an-

1 Frankel an Becker, Paris 22.II.1870 (D I 629).

2 Nach reichlich zwei Monaten Unterbrechung wieder in Soc.-Dem., Jg. VI, Nr 48 (24.IV.1870); der Zusatz ,(Mitglieder...)" ist neu, Versammlungstag ist Montag, Lokal - die Brasserie Bergrath, wo auch die deutsche Sektion tagt. Parallel dazu wird seit Nr 49 (27.IV.1870) für ,,Die Mitglieder des Allg. deutsch. Arb.-Vereins" eine Versammlung am ersten Donnerstag jeden Monats im Café Charles angezeigt.

3 Peter an Becker, Paris 27.II.1870 (D II 579).

4 O. Testut, Association Internationale des Travailleurs, S. 181.

5 Vgl. Schily an Becker, Paris 21.V.1870 (D II 984). 
scheinend auf dieser Versammlung vereinbart wurde, direkt betroffen war: Frankel bat Becker sicher nicht auf eigenes Risiko, ihm „40 Nummern des ,Vorbote' zuzuschicken, obwohl wir erst 35 Mitglieder sind". ${ }^{1}$ Was Schily später "das neue System[, das] den Kreis deiner Abonnenten vermehren muss", nannte, ${ }^{2}$ der Vertrieb der Genfer Publikationen durch die Sektion, war damit Ende Februar schon eingeführt. Zunächst bedeutete es einen sprunghaften Anstieg der Pariser Abonnentenzahl - Schily hatte im Sommer 1869 nur noch fünf Abonnenten gehabt. ${ }^{3} \mathrm{Zu}$ einer nennenswerten weiteren Expansion ist es wohl nicht gekommen, da die Mitgliederzahl, wie Frankel im Juli mitteilte, bei vierzig stagnierte. Das "neue System” fand sein Ende kurz vor dem Ende der Sektion: gegen Ende Juli waren - als Folge der gerichtlichen Auflösung der Pariser Föderation oder des Kriegsausbruchs - „von der deutschen Sektion alle Vorboten abbestellt” worden. ${ }^{4}$

Ein weiterer Schritt, den Frankel Ende Februar unternahm, wird ebenfalls kaum ohne Verabredung mit den zum organisierten Anschluss an die Assoziation entschlossenen Teilnehmern der Versammlung vom 17. II., oder mindestens der folgenden vom 24.II., erfolgt sein. Frankel nahm Kontakt mit dem Generalrat der IAA in London auf. ${ }^{5}$ Aus seiner Korrespondenz mit Joh. Ph. Becker seit Anfang Januar, vielleicht auch aus anderen Quellen, muss ihm die latente Rivalität zwischen Genf und London deutlich geworden sein. Seiner schlichten Mitteilung, er werde sich ,die Statuten von London senden lassen", " war offensichtlich Beckers prompte Aufforderung zum Anschluss an das Genfer Zentralkomitee gefolgt. Die Antwort:

„Was aber das Anschliessen an die Zentralisation der Sektionsgruppe deutscher Sprache anbelangt, wird es wohl nötig sein uns hierüber zu verständigen."7

zeigte, dass Frankel das Problem erkannt hatte; taktisch geschickt versuchte er es dadurch herunterzuspielen, dass er die eigene Ansicht hervorhob, „so wenig als möglich Zeit zu verlieren mit sogen. innern Vereinsangelegenheiten", Gleichgültigkeit in der Frage der Anerkennung der konstituierten Sektion durch London oder Genf betonte und die Absicht aussprach, „dass wir in Paris keine Ausnahme machen

1 Frankel an Becker, 22.II.1870 (D I 629).

2 Schily an Becker, a.a.O.

s Vgl. Schily an Becker, Paris 1.VII.1869 (D II 981).

4 Peter an Becker, Paris 1.VIII.1870 (D II 580).

5 Vgl. Minutes 1868-1870, S. 214.

- Frankel an Becker, 1.I.1870 (D I 627).

7 Frankel an Becker, Paris 24.I.1870 (D I 628). 
werden u. uns gleich den übrigen deutschsprechenden Sektionen an das Zentralkomitee in Genf anschliessen." ${ }^{1}$ In der eigentlichen Gründungsphase geriet Frankel die Vorsicht, mit der er die Sektion aus neuerlichen fraktionellen Auseinandersetzungen heraushalten wollte, jedoch fast zur Intrigue. Etwa gleichzeitig teilte er die Gründung der Sektion Becker mit und fragte in London an, ob die neue Sektion mit dem Generalrat korrespondieren solle. Da die Korrespondenten offensichtlich von dem jeweils parallelen Briefwechsel nicht unterrichtet wurden, ${ }^{2}$ erhielt Frankel aus London eine positive Antwort. ${ }^{3}$ Mindestens die Form, in der diese Antwort nach Genf weitergegeben wurde, konnte bei Becker den Eindruck hervorrufen, er werde von London übergangen. Der Nachdruck, mit dem ihm aus Paris versichert wurde, man halte den Anschluss an Genf und die Korrespondenz mit London für sinnvoll, ${ }^{4}$ hat ihn in seinem Protest beim Generalrat sicherlich bestärkt. ${ }^{5}$ Dieser Zwischenfall, der die Sektion bei ihrer Gründung für einen Moment auch innerhalb der Internationale in einen Konflikt zu verwickeln schien, war aber für ihre weitere Entwicklung kaum bedeutsam; für die Dauer ihrer Existenz blieb sie, wie die weiterhin rege Korrespondenz mit Becker und sporadische Erwähnung in den Protokollen des Generalrats ${ }^{6}$ zeigen, eher auf Genf als auf London orientiert.

Rein zahlenmässig rechtfertigte die Entwicklung der Sektion Frankels anfänglich höher gespannte Erwartung kaum. Mit dreissig Gleichgesinnten und hundert im Vorjahr von Eccarius erhaltenen Mitgliedskarten der Association hoffte er im Januar „eine zahlreiche Gruppe hier bilden zu können"; wird diese Aussicht damit begründet, dass „namentlich viele deutsche Bäcker hier sind, die viel Willen, aber wenig Tatkraft haben, die aber nur angespornt zu werden braucht", ${ }^{7}$ so wirkt dies wie ein Nachklang der Gebertschen Agitationsversuche. Dass die Zahl der Mitglieder nicht auf die 23 Unterzeichner des Protests vom 17. Februar beschränkt blieb, wurde schon angedeutet. Durch welche Namen, ausser dem von Victor Schily, diese Liste zu ergänzen ist, muss eingehenderer Untersuchung vorbehalten werden. Dass

1 Ebd.

2 Vgl. Frankel an Becker, Paris 22.II.1870 (D I 629), wo eine Korrespondenz mit London nicht erwähnt wird; der entsprechende Brief an den Generalrat ist offensichtlich nicht erhalten; vgl. aber Minutes 1868-1870, S. 219.

3 Vgl. Minutes 1868-1870, S. 214.

4 Vgl. Frankel an Becker, Paris 13.III.1870 (D I 630).

5 In einem PS zu seinem vom 12.III.1870 datierten Brief an Jung (Nachlass Jung 433G) zitiert Becker Frankels Formulierung wörtlich; vgl. auch Minutes $1868-1870$, S. 219.

6 Anlässlich der Durchreise von Petersen; vgl. Minutes 1868-1870, S. 223 u. 225.

7 Frankel an Becker, Paris 24.I.1870 (D I 628). 
Frankel auf schnelles Wachstum rechnete, als er Ende Februar die Gründung der Sektion mit 35 Mitgliedern nach Genf berichtete, darauf weist ausser der schon erwähnten Bestellung von vierzig Vorboten diejenige von hundert Statuten der Sektion Genf am 13. März. ${ }^{1} 100$ Mitgliedskarten im Januar, 100 Statuten im März - die Frankelschen Hoffnungen dürften hier zahlenmässig zum Ausdruck kommen. Aber auch die Obergrenze der faktischen Expansion, die weit hinter seinen Erwartungen zurückblieb und im Vergleich mit der Entwicklung der französischen Sektionen unvorteilhaft abstach, hatte Frankel mit einer der genannten Zahlen ohne es zu wissen bereits getroffen. Sein Bericht von Mitte Juli 1870 liest sich wie eine stilistisch elegantere Variation auf Geberts Klage über die „dummen Deutschländer”:

„denn 40 Mitglieder unter soviel deutschen Arbeitern ist geradezu gesagt erbärmlich. Dies hat der Deutsche (man soll keinen Nationaldünkel haben!) den Franzosen voraus, dass er immer den Schlendrian geht; Bierkneipen u. Gesangvereine sind die einzigen Elemente, die die Deutschen zusammenführen, $\mathbf{u}$. wozu ist's auch nötig, mehr zu tun, sie ist doch die jebildete Nation". ${ }^{2}$

Das Verhaltensmuster des „Deutschtums im Ausland” blieb also trotz langer vorbereitender Agitationen und trotz eines eindrucksvollen Aufschwungs der Arbeiterbewegung in der französischen Umgebung auch für die Pariser deutsche Sektion eine Grenze, die sie nicht mit nachhaltigem Erfolg überschreiten konnte. Die Gründung der Sektion darum von vornherein als einen Misserfolg zu beurteilen, ${ }^{3}$ hiesse allerdings Frankels getäuschte Hoffnung ungeteilt als Masstab des Urteils übernehmen.

\section{An allen Kämpfen der hiesigen Arbeiter beteiligt}

Unter den gegebenen Bedingungen aber konnten die Folgerungen, die Frankel aus dem Ergebnis des für das Kaiserreich günstigen MaiPlebiszits für die Agitation der Internationale in Frankreich zog, nämlich :

„dass alle Journale zur Aufklärung der Bevölkerung nicht viel nutzen, die Hälfte der Bauern kann nicht lesen, dass sie [die IAA] Agitatoren überall aussenden muss $u$. dass sie sich vom allg. $u$. direkten Wahlrecht kein radikales $u$. am Allerwenigsten ein schnel-

1 Frankel an Becker, Paris 13.III.1870 (D I 630).

2 Frankel an Becker, Paris 14.VII.1870 (D I 635).

3 Ein solches Urteil wird in enger Anlehnung an Frankels Briefe an Becker nahegelegt bei E. Silberner, a.a.O., S. $613 \mathrm{f}$. 
les Heil versprechen darf, dass der grösste Teil der Freunde des Kaiserreichs bloss mächtig sind, wenn es Stimmen zu geben heisst, dass er sich aber am Tage einer Volksforderung in den Kellern verkriechen wird, wie sie es immer getan hat. Sie hat daher bei der ersten günstigen Gelegenheit ihre Kraft überall, nur nicht bei der Urne zu fordern", 1

für die Arbeit der Sektion nur sehr begrenzt gelten. Auf den ersten Blick entsteht der Eindruck vornehmlich deklamatorischer Tätigkeit. Dem Protest vom 17. Februar folgt zeitlich der schon Testut bekannte kurze französische Aufruf in der Réforme sociale (Dokument II). Unbekannt ${ }^{2}$ war bisher dessen wenig später verfasstes, ausführlicheres deutsches Aequivalent (Dokument III). Die Geschichte dieses Dokuments deutet zugleich die begrenzten Agitationsmöglichkeiten der Sektion an, soll darum kurz referiert werden. Das Manuskript des Aufrufs übersandte Frankel Becker am 12. April mit der Bitte, „,500 Exemplare drucken zu lassen u. zwar so bald u. so billig als möglich". Am 24. April erkundigt er sich: „Wie steht's mit unseren Aufrufen?”; am 11. Mai endlich kann er den Empfang bestätigen: Becker hat die Aufrufe versehentlich an Frankels alte Adresse geschickt; dieser, der schon befürchtete, "dass dieselben mit Beschlag belegt wurden”, hat sie nur auf Umwegen erhalten. Die Verzögerung ist

„um so bedauerlicher, als wir gegenwärtig mit unsern Aufrufen wenig Propaganda werden machen können, da die Internationale sehr der Verfolgung ausgesetzt ist, $u$. bereits viele unserer Freunde unter Schloss u. Riegel sich befinden u. täglich neue Verhaftungen vorgenommen werden."

Der „III. Prozess” gegen die Pariser Internationale wirft seine Schatten voraus, Frankel selbst hat Hinweise, dass auch über ihn polizeiliche „Erkundigungen eingezogen” werden, er zeichnet diesen Brief mit dem - allerdings recht durchsichtigen - Decknamen Léo-ntine. ${ }^{3}$

Gleichzeitig mit diesem Aufruf, der unter veränderten Bedingungen sein Ziel verfehlte, hatte die Sektion ihre Existenz und Position auch Lesern von Arbeiterzeitungen ausserhalb Frankreichs bekanntgemacht. Der schon erwähnte Aufruf „An die Arbeiter Deutschlands”

1 Frankel an Becker, Paris 11.V.1870 (D I 633).

2 Jedenfalls nicht verzeichnet in Répertoire International des Sources pour l'Etude des Mouvements Sociaux aux XIX et XX $\mathrm{X}^{\mathbf{e}}$ siècles [weiter zitiert als: Répertoire], vol. III, La Première Internationale, Imprimés 1864-1876, Paris 1963 [weiter zitiert als: Bd III].

s Vgl. Frankel an Becker, Paris 12.IV.1870, und Beilage (D I 631), 24.IV. (D I 632) und 11.V.1870 (D I 633). 
(Dokument IV) erschien zunächst im Volksstaat, ${ }^{1}$ Becker vermittelte den Abdruck im Felleisen. ${ }^{2}$ Henri Bachruch gebrauchte wohl vorhandene Beziehungen, um eine ähnliche Proklamation an die ungarischen Arbeiter in Budapest veröffentlichen zu lassen (Dokument V). Ohne vorläufig auf eine inhaltliche Analyse dieser Aufrufe eingehen zu können, lässt sich aus ihrer Kombination doch mindestens schliessen, dass die Pariser deutsche Sektion ihr Zielgebiet weniger durch nationale Zugehörigkeit, eher - und dies analog zu Beckers Genfer Zentralkomitee - durch die Reichweite der deutschen Sprache als Agitationsmittel bestimmt sah.

In die gleiche Richtung zielte ein Teil der publizistischen Aktivität. Frankels ausführlicher Bericht über die Konstituiering der Pariser Föderation im April $^{3}$ unterschied sich nicht nur durch erhebliche Detailkenntnis von der bisher eher summarischen Frankreichberichterstattung des Vorbote. Auch der Vergleich mit gleichzeitigen Pariser Korrespondenzen des Volksstaat ${ }^{4}$ zeigt, welchen Vorsprung an Informationswert der Bericht des unmittelbar Beteiligten vor dem des relativ distanzierten Beobachters auch dann erzielen konnte, wenn von vollständiger politischer Übereinstimmung des Berichterstatters mit der Redaktion des Blattes nicht die Rede sein konnte. ${ }^{5}$ Voraussetzung derart unmittelbarer Beteiligung aber war, wie Frankel selbst im gleichen Bericht erläuterte, schon rein statutenmässig die Mitgliedschaft in einer Sektion. ${ }^{6}$ Solange also der Nachweis früherer Mitgliedschaft in einer französischen Sektion nicht geführt ist, ${ }^{7}$ wird man annehmen dürfen, dass Frankel als „korrespondierender Sekretär” der deutschen Sektion an den Gründungsverhandlungen der Pariser Föderation beteiligt war, ${ }^{8}$ zum Mitglied des Föderalrats gewählt wurde und als dessen „korrespondierender Sekretär für Deutschland”9 fun-

1 Volksstaat, Nr 32 (20.IV.1870)

2 Vgl. Frankel an Becker, Paris 12.IV.1870 (D I 631).

3 Frankel an Becker, Paris 24.IV.1870 (D I 632); mit unwesentlichen stilistischen Kürzungen abgedruckt in Vorbote, Jg. V, Nr 5 (Mai 1870), S. 78-80, und Nr 7 (Juli 1870), S. 110f.

4 Die Korrespondenzen im April und Mai 1870 stehen ganz im Zeichen des Plebiszits; vgl. Volksstaat, $\mathrm{Nr} 28,30$ (6. und 30.IV.) und $\mathrm{Nr} 36,38,40$ und 42 (4., 11., 18., und 25.V.).

$5 \mathrm{Vgl}$. den ausdrücklichen redaktionellen Hinweis in Vorbote, Jg. V, Nr 5 (Mai 1870), S. 78; über Hess' Verhältnis zur Redaktion des Volksstaat vgl. Silberner, a.a.O., bes. S. 609 .

- Vgl. Vorbote, a.a.O.

7 Vgl. DBMOF, Bd VI, S. 91-92.

8 Vgl. Vorbote, a.a.O.

- So unterzeichnet Frankel seinen Brief vom 12.VI.1870; ein PS, das „Nachrichten von der deutschen Sektion" enthält, ist unterschrieben „Korrespond[ierender] Sekretär der Pariser deutschen Sektion". 
gierte. Dass er seine Verwicklung in den „III. Prozess”- und man darf vermuten, einen Teil seines Prestiges in Paris - diesem organisatorischen Hintergrund verdankte, wurde dann im Juli gerichtsnotorisch. ${ }^{1}$ Im April formuliert, zum Zeitpunkt des Drucks durch den Kriegsausbruch bereits überholt, war die Art, wie Frankel die Funktion der deutschen Sektion bestimmte, dennoch treffend: „an allen Kämpfen der hiesigen Arbeiter beteiligt” hatte sie nach seiner Meinung „nicht nur eine Bedeutung für uns, weil sie aus Landsleuten besteht, sondern vielmehr weil sie ein kräftiges Mittel um die brüderlichen Gefühle der Arbeiter beider Länder ist."2

Zunächst aber, in der ersten Hälfte des Jahres 1870 , bedeutete die Teilnahme „, an allen Kämpfen der hiesigen Arbeiter” für die deutsche Sektion vor allem finanzielle Inanspruchnahme. Indiz dafür ist die Tatsache, dass Frankel Becker erst im Juli die „Abrechnung unseres Beitrags für das Zentral-Komitee" ankündigen konnte. ${ }^{3}$ Die Verzögerung musste ihm umso peinlicher sein, als er vor der Gründung der Sektion ausdrücklich erklärt hatte, man werde den „Beistand für die unterdrückten Arbeiterbrüder aller Länder" als einen Hauptzweck der Sektion betrachten, ${ }^{4}$ mit anderen Worten Becker die Kontinuität seiner Pariser deutschen Ressourcen versichert hatte. Andererseits lag gerade hier die von Ladendorf befürchtete und vorsichtig angedeutete materielle Konsequenz der Option für die französische Bewegung. Als Entschuldigung formuliert, lautet sie so:

„Die unzähligen Grèves, die an allen Seiten zu Tage treten, die Verfolgung unserer Parteigenossen von Seite unserer lieben Regierungen, wodurch oft Familien ihrer einzigen Stütze beraubt werden, legt den verschiedenen sich in Arbeit befindlichen Genossen die ernste Pflicht auf, die für unsere Sache kämpfenden Männer nicht fallen zu lassen. So kam es auch, dass unsere Sektionskasse nur ein Defizit aufzuweisen hat. Die Sache in Creuzot allein wurde von unserer Seite mit mindestens $200 \mathrm{fr}$. bedacht, nicht der andern Grèves etc. zu denken."5

Für Frankel selbst und wenigstens ein anderes Mitglied der Sektion bedeutet Teilnahme „an allen Kämpfen” zudem publizistische Tätigkeit für französische Blätter. Als sich die Pariser Föderation nach der Unterdrückung von La Marseillaise mit Le Socialiste ein kurzlebiges

1 Vgl. bes. Frankels Begründung seiner Aussageverweigerung mit der notwendigen Rücksprache mit den Mitgliedern der Sektion, III. Procès, S. 213-215.

2 Vorbote, Jg. V, Nr 7 (Juli 1870), S. 110.

3 Frankel an Becker, Paris 14.VII.1870 (D I 635).

4 Frankel an Becker, Paris 24.I.1870 (D I 628).

5 Frankel an Becker, Paris 14.VII.1870 (D I 635). 
eigenes Organ schuf ${ }^{1}$ - „von unsren Bundesratsmitgliedern redigiert”, schreibt Frankel -, erschien es selbstverständlich, dass er zur Mitarbeit aufgefordert wurde. Ebenso selbstverständlich erscheint seine Zusage; die Art, wie er sie Becker mitteilte, die Betonung der eigenen Opferbereitschaft, ist typisch für den schon vermerkten Tenor seiner Briefe aus dem Jahre 1870, sie ermöglicht aber andererseits eine kleine Korrektur seiner Biographie zu seinen Gunsten:

„Da ich gerne überall mitwirke wo man mich verlangt, nahm ich auch die Stelle als Mitarbeiter in der betreffenden Redaktion an, obwohl ich aufrichtig gesagt, da ich täglich 10 Stunden arbeiten muss zu meinem Lebensunterhalt, fast über meine Kräfte angestrengt bin."2

Einen Monat später - Frankel ist verurteilt, hat seine Haft aber noch nicht antreten müssen - gibt er Becker einen beiläufigen Einblick in die weiteren publizistischen Pläne der durch das gleiche Urteil aufgelösten Pariser Internationale:

„Dienstag erscheint wieder die Marseillaise. Der soziale Teil der früher von Millière redigiert wurde, wird von mehreren Kollektivisten der Internationalen unter einem Pseudonamen behandelt werden, unter welchen sich auch ein deutsches Mitglied befindet." 3

Henri Bachruchs frühere Mitarbeit am Travail, wie seine spätere Wahl in die Redaktionskommission von Lutte à outrance, ${ }^{4}$ legen die Vermutung nahe, der ungenannte Mitarbeiter der Marseillaise sei Frankels Nachfolger als Sekretär der deutschen Sektion gewesen. Einige etwa gleichzeitige Hinweise scheinen in die gleiche Richtung zu gehen. Sie deuten zugleich an, was mindestens für die führenden Mitglieder der Sektion Teilnahme „,an allen Kämpfen der hiesigen Arbeiter” auch und vor allem bedeuten musste: gemeinsam mit den französischen Genossen den neuerlichen Versuch des bonapartistischen Regimes, die Assoziation zu unterdrücken, abzuwehren und die Verteidigung wenn möglich zu einem Propagandaerfolg zu machen.

Gesteigerte Polizeiaktivität hatte Frankel schon in der ersten Maihälfte aus Paris berichtet und die Warnung hinzugefügt, unbekannte Zuwanderer von dort, die sich „bloss mit Karten oder Karnets” bei

1 Vgl. Répertoire, vol. I, La première Internationale, Périodiques 1864-1877, Paris 1958, S. 13 und 18.

2 Frankel an Becker, Paris 12.VI.1870 (D I 634); mindestens in dieser Phase gesteigerter Aktivität wurde Frankels Tätigkeit nicht, wie seine Biographin in vorsichtigem Anschluss an ältere Zeugnisse vermutet, durch ein privilegiertes Lohnverhältnis begünstigt. Vgl. M. Aranyossi, a.a.O., S. 16.

3 Frankel an Becker, Paris 14.VII.1870 (D I 635).

Vgl. DBMOF, Bd IV, S. 167-168. 
Becker ausweisen konnten „dreist als Mouchards [zu] betrachten”.1 Verlauf und Richtung - Anklage wegen Bildung einer Geheimgesellschaft - der richterlichen Voruntersuchung konnte er Becker einen Monat später schildern. Bestand über die Absichten der kaiserlichen Justizbehörden noch ein Rest von Unsicherheit, diejenige ihrer internationalistischen Gegner formulierte Frankel unzweideutig:

„Was die Untersuchung für Folgen haben wird, wissen wir nicht, glauben jedoch dass das Kaiserreich wie vor 2 Jahren auch jetzt für die Internationale mit seinen gehirnlosen Handlungen Propaganda zu machen."2

Als das Bekanntwerden der Anklageschrift auch die letzten $Z$ weifel über die Absichten der Kontrahenten beseitigt hatte, war es dann Victor Schily, der sich „mit gleicher Post” in inhaltlich fast völlig übereinstimmenden Briefen an Marx und Becker wandte, mitteilte, dass die Anklage auf den ,beiden Tendenzlügen société secrète u. Mazzinische Urheberschaft" aufgebaut war, ${ }^{3}$ und darum bat, ihm zu deren Widerlegung „Tatsächliches, vor Gericht Verwendbares an die Hand [zu] geben".. Dies war kaum zufälliges Zusammentreffen, eher Ausdruck dafür, dass man von Paris aus vorhandene Verbindungen gebrauchte, um die propagandistische Wirkung des Prozesses nicht nur vom guten Willen oder besser der Dummheit des öffentlichen Anklägers abhängen zu lassen. Folgt man Frankels Pariser Briefen, so war der Erfolg in den Augen der Beteiligten offensichtlich den Aufwand wert:

„Seit unserem Prozesse [...] mehren sich die Mitglieder der Pariser Sektionen, neue Sektionen bildeten sich $u$. dies sogar jetzt, nachdem der Prozess beendigt, d.h. mehrere Mitglieder verurteilt, $u$. die internat. Assoziation in Paris u. Seinedepartement für aufgelöst erklärt wurde. Da lacht Einem das Herz im Leibe, das zeigt nun Solidarität der arbeitenden Klassen". ${ }^{5}$

\section{III}

Die Darstellung befindet sich hier an einem Kreuzweg, der vorläufig durch den Namen Henri Bachruch bezeichnet werden kann. Eröffnet werden beide Wege, die - jeder auf seine Weise - zur Vorgeschichte der

1 Frankel an Becker, Paris 11.V.1870 (D I 633).

2 Frankel an Becker, Paris 12.VI.1870 (D I 634)

3 Schily an Marx, Paris 24.VI.1870 (Marx-Engels-Nachlass D 3967).

4 Schily an Becker, Paris 24.VI.1870 (D II 985).

5 Frankel an Becker, Paris 14.VII.1870 (D I 635). 
Revolution des 18. März 1871 überleiten, durch Bemerkungen Frankels, die in dem allgemein enthusiastischen Kontext des schon öfter zitierten Briefes vom 14. Juli 1870 beinahe beiläufig wirken.

\section{A German working-man}

Die eine betrifft die Sektion als selbständige politische Einheit. Direktpositive Auswirkungen des Propagandaerfolgs des Prozesses erhofft sich Frankel anscheinend nicht, die Fortsetzung der bisherigen Arbeit aber, mindestens im bescheidenen Rahmen der wöchentlichen Versammlungen, erscheint ihm gesichert - ,wird der jetzige Delegierte in Pélagie sitzen, bin ich doch überzeugt, dass ein Andrer an seinem Platz ebenso unverschrocken vorwärtsgehen wird."1 Gut zwei Wochen später ist es einer jener trocken-knappen Briefe von Philipp Peter, der Becker über die inzwischen notwendig gewordenen organisatorischen Veränderungen unterrichtet. Frankel hat seine Haft angetreten, als neue Aufgabe ist der Sektion die Betreuung der Gefangenen zugefallen: „Wir besuchen sie abwechselnd und unterstützen sie nach Kräften, bis jetzt haben sich an einem schönen Morgen im Hofe acht von Unsern begrüsst." Man versammelt sich weiter regelmässig, am ,nächsten Donnertag" will Peter die angeforderten Exemplare des Vorbote verwenden, „Bachruch ist jetzt Delegierter der deutschen Sektion”, Peter fungiert als ,Cassier”. ${ }^{2}$

Erstaunlich zu vermerken, dass vierzehn Tage nach der französischen Kriegserklärung die veränderte Situation in diesem anscheinend letzten „offiziellen” Brief eines Mitglieds der Sektion aus Paris nicht den geringsten Niederschlag findet. Geht es zu weit zu vermuten, die deutschen Internationalisten seien durch die wenig später durch die kaiserliche Regierung eingeleitete, im September durch den republikanischen Polizeipräfekten Kératry vollendete Ausweisungspolitik ${ }^{3}$ überrascht worden? Nach zwei Weltkriegen und etlichen „begrenzten Konflikten", deren keiner ohne einschneidende Konsequenzen für die organisierte Arbeiterbewegung der beteiligten Länder blieb, erscheint eine solche Vermutung gewagt. Es gibt aber Anzeichen dafür, dass Frankels Nachfolger Bachruch das ruhmlose Ende der Sektion kaum noch organisieren konnte. Peter meldet sich im November wieder bei

1 Frankel an Becker, a.a.O.

2 Peter an Becker, Paris 1.VIII.1870 (D II 580).

3 Zur Ausweisung der Staatsbürger der kriegführenden deutschen Staaten aus Paris vgl. E. Silberner, a.a.O., S. 642f.; das Ausweisungsdekret vom 6. September 1870 in: Les Murailles politiques françaises, t. I, Depuis le 18 juillet 1870 jusqu'au 25 mai 1871, Paris 1874, S. 20. 
Becker. Die Nachwirkung der Überraschung manifestiert sich bei ihm als persönliches Problem: als in Paris „alles auseinander lief”, blieb für die ordnungsgemässe Entlastung des Kassierers offensichtlich keine Zeit; die von ihm formulierte Mitteilung an die Mitglieder der Sektion (Dokument VI) war nicht nur Aufruf zur Sammlung, vor allem wollte sie wohl diesen Schritt nachholen - „dass ich gerechtfertigt dastehe." Dass dieses Dokument, das letzte offizielle Lebenszeichen einer schon nicht mehr existierenden Sektion, mit dem „patriotischen Gruss” der älteren Demokratie schliesst, den sein Autor vor- und nachher nie gebrauchte, soll hier nur notiert werden.

Ohne schon direkte Beziehung suggerieren zu wollen, sei Beckers zeitlich folgender Kommentar zu der französischen Ausweisungspolitik hier angeführt:

„Nur aus Paris hat man, laut Berichten zuverlässiger Parteigenossen über 6000 kampfbereite deutsche Republikaner hinausgeworfen. Welcher Kern deutscher Männer hätte sich angeschlossen, wenn die Republik Garantien geboten, jedweder Eroberungspolitik zu entsagen. Und wie hätte schon die blosse Existenz einer deutsch-republikanischen Legion die fürstlichen Heerhaufen [...] demoralisiert und den republikanisch gesinnten Mannschaften Anhaltspunkte und Mittel zur Erfüllung ihrer Parteipflichten geboten. Statt all' dessen..."2

Gerade wenn man Beckers betonte Distanz zur „Blauen Republik” des 4. September in den Vormonaten berücksichtigt, ist man geneigt, in dieser Formulierung die Uberraschung dessen mitzulesen, der ausgerechnet dies nicht erwartet hatte.

Überraschung darüber, wie die chauvinistische Erregung, die der Kriegsausbruch beiderseits im Gefolge hatte, die Masse der Bevölkerung den „Herrschenden” verfügbar machte, Enttäuschung darüber, wie wenig die Internationale als Organisation dieser Entwicklung effektiv entgegentreten konnte - Becker selbst hatte schon zwei Monate früher eine recht nüchterne vorläufige Bilanz gezogen:

„Auch wollen wir schliesslich noch eingestehen, dass die europäische Arbeiterpartei keineswegs die Feuerprobe der grossen Ereignisse ohne Beschädigung bestanden hat, sondern selbst noch viel zu viel von den Nationalvorurteilen des lebenden Geschlechts befangen ist."3

1 Peter an Becker, Basel 13.XI.1870 (D II 581).

Vorbote, Jg. V, Nr 12 (Dez. 1870), S. 183.

- Ebd., Nr 10 (Okt. 1870), S. 155. 
Wenn der Aspekt der getäuschten Hoffnung angesichts des Krieges hier betont wird, so darum, weil für die wenigen in Paris verbliebenen Deutschen diese Erfahrung so unmittelbar wirksam war. Schilys zutiefst deprimierter Brief nach Ende der Belagerung von Paris (Dokument IX) ist aus diesem Grunde in die Dokumentation aufgenommen. Die Voraussetzungen für die Existenz einer deutschen Sektion in Paris waren durch den Kriegsverlauf so gründlich beseitigt, dass Frankel, als er im März 1871 den im Juli des Vorjahres abgebrochenen Kontakt mit Becker wieder aufnahm, konstatieren musste:

„Sie dürfen nicht vergessen, dass sehr viele Deutsche hier lebten, die nun unmöglich hier existieren könnten, da der Hass gegen Deutsche von allen Seiten genährt wird."'

Der Kontext, in dem diese Mitteilung gemacht wurde, zeigt, dass Frankel nicht bereit war, die veränderte Situation auf sich beruhen zu lassen: Er bittet Becker, die ,intern. Arbeiterpartei in Deutschland” dazu zu bringen, ,einen Aufruf an die französischen Arbeiter [zu] erlassen". Der Gedankengang, der die Notwendigkeit einer deutschen Initiative in diesem Augenblick begründen soll, mag jenem des von Marx sarkastisch kommentierten Appells der französischen Sektionen von Anfang September ${ }^{2}$ noch verwandt sein. Die Versicherung, „Ich bürge dafür, dass [der Aufruf] von den hiesigen Sektionen im Sinne der intern. Prinzipien beantwortet werden wird", konnte der gerade in dieser Frage exponierte Frankel wohl kaum geben, wenn innerhalb des Führungskreises der Pariser Internationale der Kriegschauvinismus nicht als Problem erkannt war, seine Überwindung als notwendig betrachtet wurde.

Heisst es zu viele Elemente der Vermittlung überspringen, wenn man die hier nachgezeichnete Entwicklungslinie als empirischen Kommen$\operatorname{tar} z u$ zwei Sätzen der Marxschen Adresse betrachtet?

„Between the foreign war lost by their treason and the civil war fomented by their conspiracy with the foreign invader, the bourgeoisie had found the time to display their patriotism by organizing police-hunts upon the Germans in France. The Commune made a German working-man its minister of labour."3

1 Siehe Dokument X.

2 Siehe unten S. 134, Anm. 2, und S. 135, Anm. 1.

3 The Civil War in France, Text der 1. englischen Ausgabe, in: Archiv Marksa

i Engel'sa, Bd III (VIII), [Moskau] 1934, S. 54; vgl. dort die entsprechenden Formulierungen des ersten Entwurfs, S. 306f, und 352. Deutsch: MEW, Bd 17, S. $346,531,558 \mathrm{f}$. 


\section{Bewährte Bundesgenossen in Frankreich}

Eine zweite Linie der Darstellung lässt sich vorläufig nur als Frage formulieren: Waren durch die Arbeit der Sektion Kontakte geschaffen, die deren organisatorische Existenz überdauerten? Konnte durch solche Kontakte, als der Krieg weitergespannte Hoffnungen und Erwartungen enttäuschte, wenigstens ein Mindestmass an internationaler Kommunikation in der Krisensituation aufrecht erhalten werden? Nachweisbare Kontakte "deutscher" Internationalisten in Paris mit Johann Ph. Becker zwischen August 1870 und März 1871 sind aber - gerade im Vergleich zur Intensität früherer Kommunikation - zu sporadisch, um mehr als die Richtung weiterer Untersuchung andeuten zu können.

Der erste dieser Kontakte, ein Bericht von Bachruch aus dem gerade zernierten Paris (Dokument VIII), ist nicht nur darum interessant, weil ihm der „Blanquist” Vaillant ein „,föderalistisches” Programm hinzufügte. Die Informiertheit im Detail provoziert die Frage nach dem Berichterstatter und führt zurück zu einer zweiten beiläufigen Bemerkung Frankels vom 14. Juli 1870. Wenn dieser dort den publizistischen Höhepunkt der propagandistischen Auswertung des „III. Prozesses” ankündigt - „der letzte Prozess der Internation. wird dieser Tage in Druck erscheinen" - verwies er, ohne den Namen zu nennen, auf eine andere Aktivität seines Nachfolgers. Spätestens in der ersten Julihälfte hatte Bachruch das Zirkular unterzeichnet, mit dem die

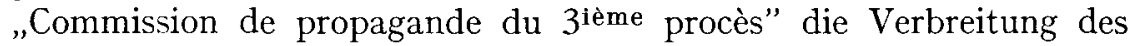
Drucks vorbereitete (Dokument VII). Die auf Juli datierte „Privatenquete" der Mitglieder der statistischen Kommission des Föderalrates, die Bachruch mitunterzeichnete, dürfte Testut, die Polizeikassandra, kaum mit gleichem Nachdruck als Beispiel der Beschränkung auf soziale Fragen gelobt haben, ${ }^{1}$ wenn ihm die partielle personelle Überschneidung zwischen beiden Kommissionen bekannt geworden wäre.

Von der Ausweisung wurde Bachruch wohl auf Grund seiner Staatsangehörigkeit nicht betroffen. Seine Name findet sich in der Folgezeit auf einer Reihe wichtiger Dokumente der Pariser Internationale. Den berühmt gewordenen Aufruf vom 4. September ${ }^{2}$ hat er nicht nur mitunterzeichnet; er war es auch, der ihn $\mathrm{Marx}^{3}$ übersandte und damit

1 O. Testut, L'Internationale et le jacobinisme au ban de l'Europe, Bd I, Paris 1872, S. $179-182$.

2 „Au peuple allemand...", Répertoire, Bd III, Nr 225; der Text wiederabgedruckt in: J. Rougerie, Paris libre 1871, Paris 1971, S. 32f.; deutsch: Volksstaat, $\operatorname{Nr} 73$ (11.IX.1870).

3 Und wohl auch Becker; vgl. Bachruch an Marx, Paris 5.IX.1870 (MarxEngels-Nachlass D 91, Fotokopie) und Dokument VIII. 
dessen schnelle und überaus scharfe Kritik auslöste. ${ }^{1}$ In jenem vertraulichen Zirkular vom September, ${ }^{2}$ das Rougerie als programmatischen wie taktischen Schlüsseltext beurteilt, ${ }^{3}$ fungiert Bachruch als Korrespondenzadresse. Im Oktober schliesslich ist er mit Hins und Leblanc Mitglied eines Initiativkomitees, das mit der organisatorischen Rekonstruktion der Pariser Sektionen beauftragt war. ${ }^{4}$ Das hier Mitgeteilte erlaubt es vorläufig nicht, Bachruchs Rolle in der Organisationsarbeit der Pariser Föderation nach deren "legaler Auflösung" näher zu bestimmen. Auch lässt sich für ihn nicht mit gleicher Eindeutigkeit wie für Frankel annehmen, dass sich diese Rolle vorwiegend aus seiner Funktion als Sekretär der deutschen Sektion herleitete. ${ }^{5}$ Immerhin ermöglichte es ihm seine Arbeit, Becker am 21. September einen Bericht über die Lage in Paris zu geben, der mindestens drei Elemente direkter Information enthält, die Becker zu diesem Zeitpunkt auf anderem Wege kaum zugänglich gewesen sein dürften. Neben dem Hinweis auf die Offizierswahlen in der Nationalgarde erscheint seine kurze Schilderung über Entstehung und Kräfteverhältnisse im Comité Central Républicain des $X X$ arrondissements als zusätzliche zeitgenössische Bestätigung der Vermutung von Rougerie über die Frontstellungen innerhalb dieses Organs, ${ }^{6}$ die Reserven, mit denen er wie Vaillant die Ergebnisse der Demarchen des Comité bei der Regierung beurteilen, ergänzen die Schilderung von Dautry und Scheler. ${ }^{1}$

Es ist nicht mit hinreichender Sicherheit festzustellen, wie diese Kenntnis Beckers Kommentierung der französischen Situation des Winters 1870-71 beeinflusste. Man ist zwar geneigt, den frühen Hinweis auf die Unfähigkeit der provisorischen Regierung, die „die eigentliche Situation Frankreichs weder begreifen [kann], noch derselben gewach-

1 Vgl. Marx an Engels, 6.IX.1870 und 10.IX.1870, MEW, Bd 33, S. 54f. und 59 f.

2 „... Dans l'impossibilité...", Répertoire, Bd III, Nr 227, Text bei J Rougerie, „Quelques documents nouveaux pour l'histoire du Comité central républicain des vingt arrondissements", in: Le Mouvement Social, Nr 37 (Okt.-Dez. 1961), S. 7f. Das Exemplar des IISG, im im Répertoire a.a.O. beschriebenen Plakatformat, trägt den handschriftlichen Vermerk: „Par erreur de l'imprimeur cette circulaire a été imprimée en affiche. Il ne faut donc pas la publier."

3 J. Rougerie, "Quelques documents", S. 7f.; vgl. auch ders., Paris libre 1871, S. $39 \mathrm{ff}$.

4 Vgl. Répertoire, Bd III, Nr 229, und den Text des Zirkulars bei J. Dautry und L. Scheler, Le Comité Central Républicain des vingt arrondissements de Paris, Paris 1960, S. $29 f$.

5 Seinen Brief an Marx, Antwerpen 19.VI.1871 (Marx-Engels-Nachlass D 92, Fotokopie) unterzeichnet er zusätzlich als „exdélegué du Cercle d'études au Conseil fédéral".

"Vgl. J. Rougerie, "Quelques documents", S. 10-12.

7 Vgl. J. Dautry und L. Scheler, a.a.O., S. 37-40 und 47-57. 
sen [ist]", " die Erwartung, „diesmal" werde im Gegensatz zu früher „die Revolution der Republik" folgen, ${ }^{2}$ wie schliesslich in der ausdrücklichen Berufung auf „zahlreiche Berichte bewährter Bundesgenossen in Frankreich", ${ }^{3}$ auch eine Resonanz der Einschätzungen Bachruchs und Vaillants zu sehen.

Andererseits widersprach die von Bachruch skizzierte Praxis und die von Vaillant angedeutete Offensivstrategie grundsätzlich Beckers eigener Auffassung von den Möglichkeiten der Arbeiterbewegung, in den Krieg der „Gewaltstaaten” handelnd einzugreifen. Man wird sein ebenfalls im Oktober formuliertes - Urteil, es sei „wahrhaft kindisch”, angesichts der veränderten politischen und sozialen Verhältnisse „die tatenreiche Stimmung von 1793 herbeizaubern zu wollen”, "wohl auch als implizite Kritik dieser Pariser Position lesen müssen.

Schilys Brief (Dokument IX) und der ausführliche Rückblick Frankels von Anfang März (Dokument X) mussten Beckers Urteil nur bekräftigen. Gab doch gerade letzterer mit dem Bericht über die Proklamation der Republik in Beauvais ein anschauliches Bild der Diskrepanz zwischen dem durch den Aufschwung in der Metropole genährten revolutionären Willen der Pariser Internationalisten und der faktischen Reichweite ihrer Agitation in der Provinz. Aber ehe sich dies in den Kommentaren des Vorbote niederschlagen konnte, ${ }^{5}$ war es schon durch die Pariser Ereignisse, eine Materialsendung Frankels ${ }^{6}$ und einen diesmal allerdings ausserordentlich knappen - Bericht von Frankel und Vaillant (Dokument XI) überholt.

Beckers erste Reaktion auf die Märzrevolution ${ }^{7}$ liest sich wie eine tastende aber treffende Synthese aus:

- dem durch den Gang der Ereignisse von „Kriegszielen” befreiten Offensivprogramm Vaillants vom September;

- den Informationen, die der Brief und die Lektüre der ersten, die Sicherung der Republik in den Vordergrund stellenden Pariser Proklamationen verschafften;

- der eigenen Skepsis, die schon jetzt das kleinbürgerliche Element in der Pariser Bewegung glaubt mitzuerkennen;

- der vorbehaltlosen Solidarisierung, ohne Rücksicht auf die Erfolgschancen.

Dass Becker zu diesem Zeitpunkt gerade auf diesen Kontakt ${ }^{8}$ grossen

1 Vorbote, Jg. V, Nr 10 (Okt. 1870), S. 152.

2 Ebd., S. 151

3 Ebd., S. 153.

4 Ebd., S. 151.

5 Die Märznummer des Vorbote erschien erst nach dem 8.IV.1871.

6 Vgl. Dokument X.

7 Vorbote, Jg. VI, Nr 3 (März 1871), S. 33-39.

$8 \mathrm{Zu}$ anderen Kontakten vgl. M. Vuilleumier, „L'Internationale à Genève et la 
Wert legte, zeigt seine dringliche Mahnung in der gleichen Märznummer: „An V. und F. in Paris: warum seit 22. März keine Nachricht?"1 Dass der Kontakt hier abbrach, und erst nach Frankels Flucht aus Paris wieder aufgenommen wurde, ${ }^{2}$ dass schliesslich gerade in der Zwischenzeit Leo Frankel seine Korrespondenz mit Marx und dem Generalrat in London intensivierte, kann vielleicht als Anzeichen einer Umorientierung interpretiert werden. Vielleicht aber war es auch einfach Ergebnis der Tatsache, dass über Brüssel die Verbindung mit London bis in den Mai hinein aufrecht erhalten werden konnte, während die direkte Verbindung mit Genf von vornherein durch die Versailler Linien lief. ${ }^{3}$

Frankels Fluchtgeschichte, ${ }^{4}$ wie deren diplomatische Weiterungen ${ }^{5}$ sind ausführlich dargestellt worden. Henri Bachruch - während der Kommune wahrscheinlich in Ungarn - war nach kurzem Aufenthalt in London ${ }^{6}$ schon im August nach Paris zurückgekehrt und beteiligte sich an den Versuchen, verfolgten Kommunarden die Flucht aus Frankreich zu ermöglichen. ${ }^{7} \mathrm{Ob}$ man in der Tatsache, dass er "mehrere alte Mitglieder der deutsch. Sektion" traf, ${ }^{8}$ schon den Ansatzpunkt zu neuerlicher Sammlung sehen kann, wird zu untersuchen sein. Ein Jahr später treffen sich drei früher führende Mitglieder der Sektion mit dem zukünftigen Sekretär des Generalrats in New York. Das Ergebnis dieses Treffens lässt, in der scherzhaft informellen Weise der Formulierung verfremdet, noch einmal die Tradition der deutschen Sektion in Paris anklingen: wenn man Becker die Teilnahme an dem für das weitere Schicksal der Internationalen Arbeiterassoziation entscheidenden Kongress finanziell ermöglichen wollte, so war man zugleich bemüht, abgerissene Verbindungen wieder anzuknüpfen (Dokument XII). ${ }^{\mathbf{9}}$

Commune de Paris (1871)”, in : Mélanges offerts à M. Paul-E. Martin, Genf 1961.

1 Vorbote, a.a.O., S. 48.

${ }^{2}$ Mit Frankels Fluchtbericht vom 12.VI.1871 abgedruckt in Vorbote, Jg. VI, Nr 7 (Juli 1871), S. 109-112, und Nr 8 (Aug. 1871), S. 121-125.

3 Zur Frage der Verbindungen vgl. u.a. die Artikel von D. Devreese und M. Vuilleumier in diesem Band.

4 M. Aranyossi, a.a.O., passim.

5 P. Agran, „Neizvestnye stranicy biografii kommunara Leo Frankelja”, in: Voprosy Istorii, 1956, Nr 3, S. 96-103.

- Vgl. DBMOF, Bd IV, S. 167f.

7 Vgl. Bachruch an Marx, Paris 16.VIII.1871 und 3.IX.1871 (Marx-EngelsNachlass D 94 und 95, Fotokopie).

8 Bachruch an Marx, Paris 16.VIII. 1871, a.a.O.

- Nach Drucklegung erschien: E. Siklós-Vincze, „Ungarn in der Kommune von, Paris und die Rückwirkung der Pariser Ereignisse auf Ungarn", in: Archiv für Sozialgeschichte, Bd XI (1971), S. 449-479; dort u.a. zusätzliche Hinweise zu Frankel und Bachruch. 


\section{DOKUMENTE}

\section{I-VI: ÖFFENTLICHE STELLUNGNAHMEN DER SEKTION}

\section{Au citoyen Barberet, secrétaire \\ de la MARSEILlaise}

Les soussignés, socialistes allemands, membres de l'Association internationale, vous prient de vouloir bien joindre leurs noms à la protestation contre l'arrestation arbitraire du citoyen $\mathrm{E}$. Varlin, en exprimant leur sympathie à ce vaillant combattant de la cause socialiste.

Paris, le 17 février 1870.

Henri Bachrutch, 12, rue de L'Echiquier; Léo Frakel, rue Saint-Sébastien, 37; L. Peterzen; Ladwig; Wytzka; Otto Tietze; Ernest Lange; Philippe Petera; Auguste Funke; T. Stade; Haubold; Stober; A. Curtaz; H. Lampe; E. Unger; L. Ratz; C. Schmidt; Edouard Ludwig; Frédéric Vollner; Jos. Werding; Otto Ludwig; Stossel; Winkl.

Zuerst in La Marseillaise, $\mathrm{Nr} 62$ (20.II.1870); unveränderter Abdruck nach L'Egalité, Genève, Jg. II, $\mathrm{Nr} 9$ (26.11.1870). Vergleich mit einem als Druckvorlage ungeeigneten Mikrofilm von La Marseillaise erlaubt folgende Korrekturen: Henri Bachruch, 13, rue ...; Leo Frankel; L. Petersen; Philippe Peters [sic].

L'Association Internationale des Travailleurs aux ouvriers allemands.

Des travailleurs allemands résidant à Paris ont pris la résolution d'adhérer au programme de l'Association internationale des Travailleurs en formant une section, convaincus qu'ils sont que ce n'est que par l'union fraternelle des travailleurs, que la classe ouvrière pourra s'émanciper de son assujettissement au capital et de la servitude morale et matérielle qui en résulte. Un motif particulier nous appelle vers cette association: c'est que son prochain Congrès doit se tenir à Paris, selon la résolution du Congrès de Bâle. Ce Congrès au profit du travail sera plus important que tous les Congrès tenus par les faiseurs politiques. Les admissions sont reçues chez Peterson, quai d'Anjou, 29; Stade, rue de la Michaudière, 3; Ratz, rue de la Victoire, 21; Bachrück, rue de l'Echiquier, 23; Frankel, (Léon), rue Saint-Sébastien, 37.

Zuerst in La Réforme sociale, Rouen, 3.IV.1870; unveränderter Abdruck nach $O$. Testut, Association Internationale des Travailleurs, Lyon 1870 , S. $180 \mathrm{f}$. 


\section{AN DIE DEUTSCHEN ARBEITER IN PARIS}

Arbeiter! In allen Städten und Ländern organisiren sich die Arbeiter und treten zu Genossenschaften zusammen, um sich gemeinsam über die Mitteln, womit das Elend des enterbten Standes, d. h. der arbeitenden Klasse beseitigt werden kann, zu berathen.

„Sparet! alle andern Rathschläge sind Hirngespinnste, womit man Euch nur den Geist zu umstricken sucht, um Euch mit Hass gegen Eure Arbeitsgeber und Fürsten, die nur allein Euer Bestes wollen, zu erfüllen" werden Euch die Einen entgegen rufen.

„Ertraget, ergeben im Herrn Euer Geschick, denn nur von kurzer Dauer ist das Dasein auf dieser Welt, und je mehr Ihr hienieden leidet, desto grössere Glückseligkeit harret Euer im Jenseits" werden Euch die Andern sagen.

Doch Heuchler sind sie Alle, die eine solche Sprache führen, denn sie Alle, Kapitalisten, Fürsten und Pfaffen, sie zehren nur vom Mark der produzirenden Klassen. Sie, die einzigen unproduktiven Arbeiter, sie haben ein gleiches Interesse Euern Geist und Eure Seele zu verkrüppeln, sie alle wissen, dass nur das Elend - die Mutter aller Leiden - allein im Stande sei den Charakter eines Volkes zu schwächen und gefühllos für „Freiheit”, „Gleichheit” und „Brüderlichkeit" zu machen.

Diese Prinzipien, welche die glorreiche französische Revolution von 1789 auf ihre Fahne schrieb, sie sind der Gerechtigkeit entstammt, welche da ruft: „Es werde Jedem das Seine" und die daher eine ewige Feindin der Lohnarbeit ist, die nur die Sklaverei verkappter Form darstellt.

Erkennt Ihr nicht, dass es nur das persönliche Interesse Eurer Unterdrücker sei, Euch in der Unfreiheit aller Art zu erhalten? Begreift Ihr nicht, dass es nur die Furcht sei, dass Ihr eines Tages - hoffen wir dass er nicht ferne sei - das Schreckliche Eurer Lage erkennen und in gerechter Wuth entbrannt, Eure Fesseln sprengen und die Lebenskraft dieser Parasiten vernichten würdet, dass die geistigen Handlanger Eurer Ausbeuter Euch immer vorschwätzen, dass Eure Lage von ewigen Naturgesetzen bedingt sei?

Man benützt diese gleissnerischen Worte nur jenen Arbeitern gegenüber als Wiegenlied, die kindisch genug sind sich damit noch einlullen zu lassen. Doch jenen Männern der Arbeit, welche diese Heuchelei bereits durchschaut haben, und sich mit Abscheu abwendend mit um so lauterer Stimme nach ihren Rechten fordern, jenen Männern die eine soziale eine politische Gleichstellung für Alle, ohne Unterschied des Geschlechts, des Glaubens und der Nation wollen, wird mit Gefängniss und nicht selten mit Pulver und Blei geantwortet. Blickt um Euch her und Ihr werdet die Belege dafür in derNiedermetzlung der Arbeiter in Seraing, Aubin, Ricamarie und Swarow sowohl, als in den Verhaftungen in Wien, Creuzot, etc., finden.

Diese Akten roher Gewalt, welche der Sklavenzeit entlehnt, Euern Brüdern gegenüber, die kämpfend für sich zugleich die Sache aller Arbeiter verfechten, in Anwendung gebracht werden, entreissen sie Euch nicht der Lethargie, in welcher Ihr fast schon zu lange gelegen? ermannen sie Euch nicht, und rufen Euch zur Pflicht mit einzutreten im Kampfe für die Rechte der Arbeiter?

Wenn Ihr Euch nur ferne von der Fahne der Sozialdemokratie haltet, weil Ihr von den Gedanken umfangen seid, dass Ihr als Söhne Deutschlands für die französische Arbeiterbewegung weder zu wirken braucht noch dürft, dann befindet Ihr Euch in einem bedauerlichen Irrthum. Die Befreiung der arbeitenden 
Klassen von sozialen wie von politischen Joche, ist keine Aufgabe deren Lösung an Sprachen gebunden ist, sie ist vielmehr eine internationale, $d . h$. eine allen Ländern gemeinsame Aufgabe, die daher auch nur durch die Verbindung der Arbeiter aller Länder gelöst werden kann.

Aus diesem Bedürfniss ist eben die internationale Arbeiterassoziation, die bereits in allen Ländern verzweigt ist, und Millionen von Mitgliedern zählt, hervorgegangen. Dieser Assoziation, welche die Arbeiterbewegung in allen Ländern mit allen ihr zu Gebot stehenden Mitteln unterstützt, beizutreten, halten wir als die Pflicht eines jeden Mannes der für die soziale und politische Gleichstellung Aller in die Schranken treten will.

Die deutsche Sektion der internationalen Arbeiterassoziation in Paris.

N.-B. - Beitrittserklärungen werden jeden Donnerstag von 8 bis 11 Uhr Abends im obern Saale der Brasserie, Rue de la Tacherie, 7, entgegen genommen.

Genf. - Cooperativ-Buchdruckerei, Rue de Carouge, 6.

Ms. mit geringfügigen orthografischen Abweichungen, zweieinhalb Seiten von der Hand Frankels als Beilage zu Frankels Brief vom 12.IV.1870 im Nachlass Becker (D I 631); unveränderter Abdruck nach dem Flugblatt in IISG, 2 S., $21 \times 14,5 \mathrm{~cm}$, s.d. [zwischen 12.IV. und 11.V.1870], Int $175 / 36 \mathrm{R}$.

\section{IV}

An die Redakton des „Felleisen["] in Zürich.

Geehrte Redaktion! Ich ersuche Sie in der nächsten Nummer folgenden Aufruf gefälligst aufnehmen zu wollen.

\section{AN DIE ARBEITER DEUTSCHLANDS!}

Arbeiterbrüder! Ueberall, wohin wir auch unser Auge wenden mögen, sehen wir die Arbeiterklasse unter dem Doppeljoche einer industriellen wie politischen Tyrannei sich keuchend um ihr karges Leben abmühen.

Von allen Seiten schlagen Verzweiflungsrufe an unser Ohr und verzweifelnd müssen wir uns fragen, wie lange es noch währen soll, dass des Volkes Gut und Blut, des Volkes Kraft und Leben die Beute unersättlicher Vampyre sei.

Der Mann, der dem Klima und der Witterung trotzend die Erde der ganzen Gesellschaft nutzbar macht, der die Sümpfe trocken legt und so die Luft von Miasmen reinigt; der Mann welcher in die Tiefen der Bergschachte steigt, um Erz und Kohlen an's Tageslicht zu fördern; der Mann, der den Stürmen und den Wellen trotzend dem Meere die verborgenen Schätze entlockt; der Mann endlich, der alle Reichthümer hervorbringt, - soll $\mathrm{ihm}$ stets das Elend sein Wiegenlied, das Elend sein Grabgesang sein? Soll er stets nur Kapitalien schaffen, um einigen Gewaltshabern noch mehr Macht über ihn zu herrschen in die Hände zu geben? Soll er stets nur der Schöpfer seiner eigenen Mordwerkzeuge sein?

Arbeiter! Je geduldiger wir es geschehen lassen, dass man uns in jeder Weise ausbeutet, desto muthiger, desto häufiger werden uns die Schläge treffen. Die Hinmordung unserer Brüder in Searing[!], Ricamarie, Aubin und erst kürzlich in Swarow, die Verhaftung unserer österreichischen Leidensgefährten, die nun schon seit Monaten in den Kerkern Wiens schmachten, das durch die Hart- 
näckigkeit einiger industriellen Feudalherren hervorgebrachte Elend in Waldenburg, die Aushungerung unserer Brüder in Irland, sind sie nicht die untrüglichsten Belege hiefür? - Sollten wir aber deshalb verzagen, oder gar uns demüthig zu den Füssen unserer Gebieter legen?

Nein! Nein! denn alle diese Leiden sind nur Wolken, die sich am sozialen Himmel lagern, und, täuscht uns nicht der oft heroische Widerstand unserer Brüder, sind dies Blitze, die ein nahes Gewitter verkünden, welches je mächtiger, je weiter es über die Länder hinrollen, uns um desto sicherer von der uns schier erstickenden sozial-politischen Schwüle befreien wird. Alle die Streiche, welche man gegen die Arbeiter führt, um dieselben in Furcht zu jagen, bilden im Gegentheil ebenso viele neue Korps, die nur den Moment abwarten, um auf dem Kampfplatz erscheinen zu können. Alle die vereinzelten Widerstandsleistungen gegen die Anmassung der Kapitalisten sind Plänklergefechte, die der grossen Schlacht vorauseilen. Soll aber diese Schlacht eine siegreiche werden, müssen besonders die dem Schlachtfelde zunächststehenden Truppen unterstützt werden.

Gegenwärtig stehen die Arbeiter Creuzot's im Feuer, und betrachtet man Frankreich als den geeignetsten Kampfboden, um unsere Feinde niederzuschmettern, ist es auch die erste Pflicht, denselben beizustehen. Dieselben haben schon seit Wochen ihre Arbeit eingestellt und die Energischsten von ihnen, die sämmtlich Familienväter sind, wurden zu mehreren Jahren Kerker verurtheilt. Es hat daher Jeder, der zur sozial-demokratischen Fahne gehört, eine um so ernstere Aufgabe zu erfüllen.

Arbeiter, Ihr kennt Eure Pflicht!

Mit sozial-demokratischem Brudergruss

Die deutsche Sektion d. intern. Arbeiterassociation in Paris. Leo Frankel, corresp. Sekretär.

Rue St. Sébastien 37.

NB. Geldsendungen werden bereitwilligst von dem Unterfertigten wie von der Redaktion der „Marseillaise”, Rue d'Aboukir 9, zur Weiterbeförderung entgegengenommen.

Zuerst in Der Volksstaat, Nr 32 (20.IV.1870) mit einer Reihe von stilistischen Abweichungen, Anschreiben und der Zusatz „NB. [...] entgegengenommen" fehlen; unveränderter Abdruck nach Felleisen, ... Zürich, Jg. IX, Nr 17 (23.IV.1870) Frankel hatte in seinem Brief vom 12.IV.1870 Becker um die Weiterleitung des Aufrufes an die Redaktion dieses Blattes gebeten.

\section{V}

\section{MANIFEST}

Die Pariser Arbeiter an die Arbeiter Ungarns.

Arbeiter!

Mit Freude vernahm die Pariser deutsche Sekzion, dass die Prinzipien der Internazionalen Arbeiterassoziazion nunmehr auch bei Euch anfangen, Boden zu fassen und dass auch Ihr gewillt seid, bald mit der allgemeinen sozialen Bewegung mitzumarschiren.

Ueberall an allen Orten, in allen Staaten raffen sich die seit Jahrhunderten in Knechtschaft und im Elend verkümmernden Massen auf und lassen ihren Schrei 
nach Gerechtigkeit und Freiheit ertönen; ohne Unterschied der Nazionalitäten reichen sich die Arbeiter aller Länder die Bruderhand und organisiren sich, um die Mittel ihrer ökonomischen und politischen Befreiung zu suchen, in Genossenschaften der einzelnen Gewerbe, und in der leztere alle umfassenden internazionalen Arbeiterassoziazion.

Die Internazionale, welche das Programm: „Dem Arbeiter den vollen Ertrag seiner Arbeit" auf ihre Fahne schreibt, hat in allen Gauen Europa's und Nordamerika's ihre Anhänger und zählt ihre Mitgliederzahl nach Millionen.

Arbeiter Ungarns! Ihr werdet gewiss nicht zurückstehen; Eure deutschen und französischen Brüder erwarten Euren Eintritt in die Vereinigung. Ihr, die Ihr durch Eure Zahl den Staat bildet, die Ihr mit Eurem Schweisse die Gesellschaft erhaltet, welch' leztere keinen Tag ohne Eure kollektive Arbeit bestehen könnte, werdet durch die heutigen Lohnverhältnisse im Elende erhalten; Ihr seid preisgegeben der Ausbeutung einer an Zahl geringen Klasse, der Bourgeoisie, welche mit dem Kapitale bewaffnet Euch in ihrer Abhängigkeit hält.

Aber auch der mit kleinem Kapitale arbeitende Produzent wird in dies Abhängigkeitsverhältniss getrieben. Das Kapital, der Grund und Boden inbegriffen, konzentrirt sich nach und nach in immer wenigeren Händen und sammelt sich zum Grosskapital und Grossgrundbesiz an; von der Kleinbourgeoisie werden, wie leicht zu beobachten ist, immer mehr und mehr durch den kapitalistischen Entwicklungsprozess von ihrem Besize sozusagen enterbt und in die Klasse der Lohn arbeiter gestossen, denn dem kleinbäuerlichen und kleingewerblichen Produzenten wird es unmöglich, mit den Grossproduzenten zu konkurriren, welche durch Anschaffung von neuen Maschinen und verbesserten Werkzeugen, billiger en gros erkauftes Rohmaterial und weitere Ersparungen, die durch Massenerzeugung erzielt werden, im Vortheile sind.

Zahlen beweisen am besten:

In England, wo die Leibeigenschaft schon im 14. Jahrhundert aufgehoben wurde, bestand im 15.-16. Jahrhunderte die Bevölkerung hauptsächlich aus freien wirtschaftenden Bauern; die Zahl derselben betrug im

Jahre 1690 noch $82 \%$, also mehr als 4 Fünftel der Totalbevölkerung,

" 1770 blos

" 1846 blos mehr

". 1870 sollen selbe auf

250.000 Grundeigenthümer,

herabgeschmolzen sein.

30.000

18.000

Im Jahre $1863 \mathrm{gab}$ es in England 3000 Personen, die zusammen ein jährliches Einkommen von sechshundertfünfundzwanzig Millionen Frcs. unter sich theilten, mehr als das Totaleinkommen der Gesammt-Ackerbaubevölkerung von England und Wales. Die Landaristokrazie Grossbritanniens, 5000 Mitglieder zählend, hatte 1868 ein Einkommen von zwei Milliarden siebenhundertfünfzig Millionen Francs; im Durchschnitte für den Einzelnen fünfmalhundertfünfzigtausend Francs.

Im Gegensaze dazu mussten zwei Millionen achtmalhundertfünfundneunzigtausend Arbeiter-Individuen im selben Jahre in England sich mit derselben Summe abfinden, was also 950 Frcs. für den Einzelnen macht. Ersterwähnte 5000 Grundbesizer konnten das Einkommen verprassen von sechs Millionen männlichen und sechs Millionen weiblichen Ackerbauarbeitern Frankreichs, die nach der offiziellen Statistik Moreau de Youne's [sic] zusammen 2700 Millionen Francs Salair einnahmen.

In Frankreich beträgt der Werth des Ackerlandes circa 50 Milliarden Francs. Darauf schulden die Kleingrundbesizer circa 14 Milliarden Francs in Hypotheken; also mehr als der vierte Theil ist nur mehr dem Namen nach Eigenthum 
der ihn bebauenden Landbesizer, und arbeitet ein solcher „Eigenthümer” fast blos für den Parasiten, welcher als Hypothekargläubiger seine 5\% erhält, wenn er einen Bodenertrag von $7 \%$ erringt. Solcher Eigenthümer gibt es zwei ein halb Millionen und verdienen selbe circa 90 Frs. im Durchschnitte, müssen daher in der Zwischenzeit sich als Taglöhner engagiren.

Im Jahre 1789 waren von 26 Millionen Einwohnern Frankreichs

17 Millionen Lohnarbeitervolk $=65$ pCt. der Bevölkerung und

9 , andere Klassen $=35$, ," ,

Im Jahre 1850 waren von 36 Millionen Einwohnern

$30 \frac{1}{3}$ Millionen Lohnarbeitervolk mit Familien $=84 \mathrm{pCt}$. und

$5 \frac{2}{3} \quad$ andere Klassen $=16 \mathrm{pCt}$.

Es verminderte sich also die Zahl der besizenden Klasse um mehr als 55 pCt., während die Lohnarbeiterklasse - ebenfalls proporzionell sich um 20 pCt., also um ein Fünftel, vermehrte.

Arbeiter! Was ist Euer?!! Elend und Krankheit, Mühen und Sorge um's tägliche Brod, welches Euch eine Laune der Arbeitgeber, eine verfehlte Spekulazion entziehen kann; denn Ihr könnt täglich aus der Arbeit entlassen werden, und sieht man die Erbärmlichkeit der ohne Euch gemachten Gesezgebung aller Staaten daran, dass ein kleines Häuflein Parasiten, geschüzt durch die bewaffnete Macht, das Recht besizt, Massen des Volkes dem Hungertode preiszugeben! -

Wer wagt dies zu leugnen?

Die Euch unterdrückende Regierung geht Hand in Hand mit der Euch ausbeutenden Bourgeoisie und der das Volk in Dummheit und Ignoranz haltenden Geistlichkeit aller Konfessionen.

In diesen drei vereinigten Grazien habt Ihr das Bild der Reakzion!

Euren Forderungen um eine geringe mässige Verbesserung der Löhne antworten die Fabrikanten durch Pulver und Blei der Soldaten; blicket nach den Arbeitermezeleien in Brünn und Reichenberg, nach denen von Ricamarie, Aubin, Creuzot, Fourchambault in Frankreich, nach Seraing, Frameries in Belgien u.s.w.!

Die Männer, die Euch die Wahrheiten vor's Auge stellen, werden in's Gefängniss gesteckt: denn - die Eulen, Ihr wisst es ja, können das Tageslicht nicht vertragen.

In Unwissenheit erhält man Euch, denn die unwissende Masse kann sich über die sozialen Verhältnisse nicht aufklären, und sich keine Rechenschaft geben über die schamlose Ausbeutung, der sie unterliegt.

Eure Ausbeuter fürchten, dass Ihr eines Tages - hoffen wir ihn bald zu sehen das Schreckliche Eurer Lage erkennen und in gerechter Wuth entbrannt, Eure Fesseln sprengen werdet, und auf den Trümmern der heutigen, auf Kampf und Raub begründeten Gesellschaft eine Gesellschaft, welche zur Basis die soziale Gleichheit und zum Prinzipe die Gerechtigkeit hat, aufbaut.

D'rum vorwärts! Eure Pflicht ist es, in die Sekzionen und Genossenschaften der Internazionalen einzutreten, denn die „Internazionale” unterstüzt die Arbeiterbewegung mit allen ihr zu Gebote stehenden Mitteln; durch die Internazionale werdet Ihr siegen in dem Kampfe um die soziale und politische Gleichstellung.

Ungarische Arbeiter! Vorwärts!

Im Auftrage der Pariser deutschen Sekzion der Internazionalen Arbeiter-Assoziazion:

Paris, im Mai 1870. Henry Bachruch, Sekretär.

Unveränderter Abdruck nach Allgemeine Arbeiter-Zeitung, 
Jg. I, Nr 10 (5.VI.1870); für die Beschaffung der Vorlage danke ich Dr Edit Siklós-Vincze (Budapest).

\section{VI}

Hiermit thue ich den Mitglieder der deutschen Section international meinen Aufenthalt zu wissen, indem wir in die ganze Welt zerstreut sind und ich im Besitz unserer Sache bin, jetzt sowie später nach verlauf vom Krieg wieder für unsere Sache einstehen werde, und bitte die Mitglieder mit mir zu correspondiren.

\section{mit Patriotischem Grus der Cassier der deutschen Section Paris \\ Philipp Peter Spahlenvorstadt N: 19, Basel}

Unveränderter Abdruck nach dem Ms., inseriert im Brief Peter an Becker, 13.XI.1870 (D II 581). Vorbote, Jg. V, Nr 11 (Nov. 1870), S. 176 enthält lediglich eine Adressenmitteilung mit der offensichtlich falschen Angabe „Spahlenvorstadt Nr. 13".

VII-XII : MITGLIEDER DER SEKTION IN PARIS 1870-1872 (AUSWAHL)

\section{VII}

Citoyen,

Les membres de la Commission de propagande du $3^{\text {ième }}$ procès de l'Association Internationale faisant imprimer le dernier procès des Sections parisiennes vous demandent, Citoyen, de vouloir bien nous indiquer le nombre de volumes (Con̂t 1 fr. 50 Cent franco), dont vous pourriez avoir le placement dans votre groupe. Veuillez nous envoyer vos demandes le plus tôt possible et vous organiser pour en placer le plus grand nombre afin de donner une grande extension à cette œuvre de propagande socialiste.

Pour les envois d'argent,

Pour la commission de propagande.

Le Correspondant,

Henry Bachruch,

13 , rue de l'Echiquier.

S'adresser au Trésorier

Delahaye,

58, rue des Amandiers.

[en marge]

Assoc. d'Ouv. Lith. Schmit \& Cie quai Valmy, 21

Unveränderter Abdruck nach dem lithographierten Zirku-

lar im IISG, 1 S., $20,5 \times 14 \mathrm{~cm}$, s.d. [ca. Juli 1870], Int

$175 / 39$. 
VIII

H. BACHRUCH UND ED. VAILLANT AN JOH. PH. BECKER

[Stempel:]

Association Internationale

des Travailleurs

Conseil Fédéral Parisien

Paris den 21 Sept 1870

Bürger Ph. Becker Genf.

Hoffentlich haben Sie meine Postsendungen wie Manifeste erhalten und alle Schritte gethan die nur irgend für unsere Situation nützlich sein konnten.

Wie Sie wohl erfahren, haben wir in den verschiedenen Quartiers Comités wählen lassen die durch ihre Centralisation die Municipalität oder Comm. vertreten. Ob wir Recht oder Unrecht hatten uns in dieser Kriegsaffaire einzulassen und Politik zu treiben will ich dahingestellt sein lassen. Nur soviel ist zu bemerken dass sich eine Menge Republikaner im Anfange unserer Initiative bedienten und sich in den Comités derart einmischten dass wo selbe durch die Internationale ihr Prestige und Einfluss erhielten, jetzt die Interessen unserer Association nicht zu vertheidigen denken.

Wie gesagt, ich hasse dieses Republikanische Journalisten $u$ Bourgeois Gesindel; leider haben selbe eine phrasenhaftere und dem Volke angenehmere Zunge als unsere Parteigenossen.

Bitte mir gefl. Nachrichten über die Zustände in Deutschland geben zu wollen über die Arrestation des Braunschweiger Comités, über das Benehmen des Herrn von Sweizer und der Lassalleaner gegenüber der französischen Republik über das Benehmen der baierischen Sozialdemokraten, [S. 2] der Mendeschen Partei und die Stimmung der gesammten Arbeiterklasse in Deutschland.

Nachrichten über die Partei in Osstereich und Ungarn? Erscheint die allgem. Arbeiterzeitung?

All unsere Parteigenossen hier sind in die Nationalgarde aufgenommen. Wir brachten die Wahl von mehreren Officieren durch. - Varlin, Cyrille, Casse, Jaclard, Vallès, commandiren jeder ein Bataillon von 1500 Mann ausserdem haben wir noch circa 20-30 Capitaine unserer Partei können deshalb energische Massregeln gegenüber der Reaction nehmen. Die provisorische Bourgeoisregierung ist wie es zu erwarten war ganz unfähig die Geschäfte zu leiten. Das Central Comité hat von der Regierung die Ausführung der Beschlüsse die in den 20 Arrondissements gefasst wurden verlangt und[?] schlecht empfangen; und entschliesst sich die Regierung all das $z u$ thun so thut sie es verkehrt, ihre Entschuldigung ist immer die Beschäftigung mit der Vertheidigung von Paris.

Paris ist cernirt und weiss ich nicht ob Ihnen lieber Bürger diese Zeilen in die Hände kommen. Vorgestern fand ein Kampf bei Meudon Clamart statt der für uns schlecht ausfiel.

Die niederträchtigste Unfähigkeit.

Mit sozialdemokratischem Grusse und Handschlage Henry Bachruch.

[S. 1, linker Rand:]

Amitiés cordiales. Rien n'est perdu. La marche des Prussiens sur Paris nous a donné la forme republicaine; leur arrivée sous nos murs nous donnera peut etre 
la Revolution. Nous voulons nous defendre parceque le parti qui sauvera le pays sera maitre de la situation; mais nous ne negligeons pas l'ennemi interieur mille fois plus redoutable. Pas de constituante. La commune de Paris; communes revolutionnaires partout, federées. Enfin bon espoir, et puissions nous nous revoir bientot dans de meilleures circonstances la Republique consolidée. Mes compliments à $\mathbf{M}^{m e}$. Becker. Amitiés cordiales à Mme. Wilhelmi et à Bertha je les embrasse de tout cœur. En toute hate je vous serre fraternellement la main. Vive la Republique sociale! Votre ami devoué

Ed. Vaillant

Nous n'avons pas encore de reponse définitive du gouvernement provisoire à notre mise en demeure. Il ne peut y faire droit, si le peuple a quelque chose dans la tete et le cour sur ce terrain nous engagerons la bataille et triompherons.

Unveränderter Abdruck nach dem Original im IISG, 2 S., $27 \times 21 \mathrm{~cm}$, Nachlass Becker D I 28

IX

VIKTOR SCHILY AN JOH. PH. BECKER

[Nach der Unterzeichnung des

Präliminarfriedens am 26.II.1871]

Mein lieber Alter

Ich bin in meiner Gesundheit, Stimmung etc. noch zu sehr angegriffen, um dir ausführlich schreiben zu können. Von den horreurs du siège, schmale u. schlechte Kost, etc., war mir jedoch nicht das physische Element (vertreten namentlich durch ungeniessbares Brod) das Schrecklichste, sondern der schrecklichste der Schrecken war für mich der franz. Chauvinismus, wie er sich in der hiesigen Presse kund gab, durch die lächerlichste Ueberhebung der grrrrande nation $u$. die gemeinste Herabsetzung [S. 2] des deutschen Volkes, in allen seinen Theilen, ohne Unterschied zwischen Regierern u. Regierten. Wenn ich die Kraft dazu finde, habe ich vor Etwas zu schreiben unter dem Titel z.B. La vérité aux Français par un allemand, membre de l'association internationale, $u$. darin auszuführen: dass das franz. Volk (namentlich die Arbeiter) es nicht verstanden, sich dem deutschen Volke behufs Ueberwältigung der Kriegsparthei zu nähern, dass der Chauvinismus die Domäne der herrschenden Klasse in Frankreich ist, eigentlich nur die Selbstvergötterung der Litteraten, Pyaten[?], Bourgeois [S.3] aller Art, während das Volk sich nur in diese albernen Ideen hineinreissen lässt, um am Ende die Zeche zu zahlen, jetzt die 5 Milliarden; etc., etc.; dass die offizielle Schichte, die jetzt wie ein"Mehlthau über dem französisch. Volke lastet, die Tagespresse, die chauvinistische Bande, vernichtet werden muss, um dem wahren Volke zum Durchbruch zu verhelfen.

In Eil, Dein

Unveränderter Abdruck nach dem Original im IISG, 3 S., $13,8 \times 11 \mathrm{~cm}$, Nachlass Becker D II 992 
Geehrtester Bürger und Bundesgenosse!

Mit gebeugten Muth nehme ich die Feder zur Hand um die so lange unterbrochene Correspondenz wieder aufzunehmen.

Oftmals schon begann ich ein Schreiben welches ich aber wieder unbeendigt bei Seite legen musste da sich die Feder sträubte meine Gedanken wieder zu geben. Ob es mir heute gelingen wird, noch weiss ich's nicht zu sagen, denn kaum lasse ich alle die Ereignisse welche ich seit einigen Monden durchlebte an mein geistig Auge vorüberziehen wird es mir schwer um's Herz, meine Gedanken umwölken sich und ich halte dann nur noch mechanisch die Feder in der Hand.

Wer an meiner Stelle könnte auch muthiger sein? Zeit, Geld, Freunde, Verwandte, die süssesten Bande, welche sonst den Menschen an das Dasein knüpfen, alles was gewöhnlich die Irdischgebornen als ein Glück betrachten ich stiess es kalt von mir zurück. Ich bemitleidete diejenigen meiner Freunde, die an sich selbst nur denkend, am Brombeerstrauch des Lebens sich festklammerten, um während des Pflückens [S. 2] die übrige Menschheit ihrem traurigen Schicksal zu überlassen, und nun welcher Lohn?

Wem soll nun heute nach den traurigen Ereignissen die sich vor den Augen der ganzen Welt abwickelten nicht $Z$ weifel aufsteigen bei dem Gedanken an die Möglichkeit einer Vervollkommnung der menschlichen Gesellschaft? Was nützen alle Wissenschaften, wenn sie nur Schurken dienlich sind? Was Chemie u. Physik wenn sie nur zur Vervollkommnung mörderischer Waffen dienen? Was Mechanik, was alle industriellen Erfindungen wenn sie nur zur Unterjochung der grossen Mehrheit beitragen? Was alle Philosophie, wenn man sie ungestraft mit Füssen treten kann? Wer könnte da nicht ein Misantrop werden und sich bloss nach irgend einem Winkel sehnen um ferne von dem Geräusch der Welt sein Dasein zu vollbringen?

Nach vierwöchentlichen Aufenthalt in St. Pelagie wurde ich mit meinen übrigen Parteigenossen nach dem Gefängnisse nach Beauvais in einem Zellenwagen abgeführt. Wir blieben jedoch nur einige Tage daselbst, da uns durch die Proklamirung der Republik in Paris den 5 September die Thüren geöffnet [S. 3] worden. Trotzdem schon ein Tag vergangen war seitdem die Republik verkündet war, wusste man noch nichts davon in Beauvais. Wir zogen nach dem Stadthause, der Bürgermeister war abwesend, da proklamirten wir selbst die Republik, liessen die Adler vom Stadthause abnehmen, hielten Reden an die Volksmenge, die ganz verblüfft dastand $u$. nicht wusste, wie ihr geschah. Unser Ruf: Es lebe die Republik! fand keinen Wiederhall, es schauerte mich. Zum erstenmal empfand ich den gründlichen Hass $\mathbf{u}$. Verachtung gegen diese Kreatur die sich der „Retter” der Gesellschaft nannte. Zwanzig Jahre seiner Regierung genügten um ein Volk unempfindlich zu machen bei dem Zerbrechen seiner Ketten. Es war während seinem Regiment derart an die Finsternis gewöhnt worden, dass es sich unheimlich fühlte bei den ersten Strahlen der Freiheitssonne. Gedankenschwer fuhr ich nach Paris zurück. Robin reiste nach Brüssel wo sich seine Familie befand. Sollte ich bleiben? sollte ich Frankreich verlassen? - Ich hatte mich unter dem Kaiserreich dem Pariser Proletariat angeschlossen, sollte ich es nun 
in den Stunden der Gefahr verlassen? Ich war überdies sozusagen der einzige Vertreter der deutschen Sozialdemokratie - die deutsche Sektion, die ich mit vielen Muihen [S. 4] in's Leben rufen half, bestand nicht mehr - da war kein Zaudern möglich, ich blieb.

Ich war allerdings nicht entzückt von dieser Republik, denn bald darauf schrieb ich im "Courrier français" die verschiedenen Aufrufe der intern. Association ins Auge fassend einen Aufsatz welcher mit den folgenden Worten schloss : [., Tandis que Guillaume et Napoléon voient dans les républicains de France leurs ennemis communs tous les peuples sont prêts à s'entendre pour acclamer la République, et pour sceller contre les rois une alliance fraternelle.

Mais la République que les peuples veulent acclamer ce n'est pas la République nominale et aristocratique dans laquelle les instruments de travail resteraient entre les mains d'un petit nombre, mais la République bassée sur le travail et l'égalité, la République démocratique et sociale."

Dessen ungeachtet schlug ich mich zur Partei der Franzosen, unterstützte die Ansichten eines Kampfes auf Tod u. Leben, wie Sie aus einem Aufrufe welchen ich Ihnen beisende, ersehen werden, denn eine gewaltige Niederlage der preussisch-deutschen Armeen vor Paris bedeutete für mich eine deutsche Republik; u. wir hätten damit ein der [S. 5] Welt nie gesehenes Schauspiel geboten; einen Krieg der im Interesse der Fürsten von denselben begonnen im Interesse der Völker beendet worden wäre. Dass es anders wurde, ist weder in dem Patriotismus der Deutschen, noch in deren numerischen Überlegenheit der Armee der Grund zu suchen, sondern einzig $u$. allein in der franz. Regierung die weil sie die Reaktion fürchtete, die Republikaner ins Gefängnis warf. Ach, ich könnte Ihnen all die Fehler zeigen, die es klar machen würden, dass wenn Frankreich in dieser traurigen Lage gekommen ist, es bloss die Schuld der kaiserl. Partei war, die nun zerrissen ist $u$. der Bourgeoisparthei die leider noch existirt. Die soziale Arbeiterparthei hat vielleicht nicht einstimmig genug gehandelt, aber wenigstens bewiesen dass sie unschuldig ist an den Handlungen der einen, wie der andern Regierungsparthei. Der 31. Oktober 1870 hat dies bewiesen.

Lassen Sie mich werther Bundesgenosse mein Schreiben schliessen, da es mir unmöglich wird dasselbe fortzusetzen. Ich bitte Sie nur noch um die internation. Arbeiterparthei in Deutschland frei zu machen von den Siegen Deutschlands, denn meines Erachtens hat Deutschland mehr Arbeit um seinen Sieg fortzuwaschen, als Frankreich [S. 6] seine Niederlage - möge dieselbe auf Ihre Anlassung hin einen Aufruf an die französischen Arbeiter erlassen, denn Sie dürfen nicht vergessen, dass sehr viele Deutsche hier lebten, die nun unmöglich hier existiren könnten, da der Hass gegen Deutsche von allen Seiten genährt wird. Ich bürge dafür, dass derselbe von den hiesigen Sektionen im Sinn der intern. Principien beantwortet werden wird.

Wenn Sie vielleicht geehrter Bürger in Correspondenz mit den Wiener Parteigenossen stehen, dann bitte ich Sie denselben meine besten Grüsse zu senden, $\mathbf{u}$. ihnen zu sagen, da es mir vorläufig unmöglich ist an ihrem Blatte mitzuarbeiten. Wenn ich vielleicht wieder Arbeit finden werde in irgendeinem Atelier werde ich auch wahrscheinlich mehr Ruhe haben um schreiben zu können. - Vielleicht ist's auch möglich dass ich Paris verlasse, trotz dem Drängen meiner Pariser Freunde hier zu bleiben es ist dann möglich dass ich nach Wien komme.

Seien Sie herzlichst gegrüsst von Ihrem Ergebenen

Leo Frankel

Unveränderter Abdruck nach dem Original im IISG, 37. Rue St. Sébastien $6 \mathrm{~S} ., 21 \times 13,5 \mathrm{~cm}$, Nachlass Becker D I 636 


\section{FRANKEL UND ED. VAILLANT AN JOH. PH. BECKER}

[Kopfbogen :]

MINISTERE de l'Intérieur

CABINET du Ministre
Paris le 22 Mars 1871

\section{Bürger J. Ph. Becker Genf}

\section{Geehrtester Bundesgenosse!}

Ich weiss nicht ob Sie die Zeitung, welche ich Ihnen vor einigen Tagen überschickte erhalten haben. In diesem Falle werden Sie schon Kenntniss von den hiesigen Vorgängen haben, Vorgänge wie sie die Geschichte bis jetzt noch nicht aufzuweisen hat und die so unvermuthet $\mathbf{u}$. plötzlich kamen, dass die gesammte regierende Welt mit ihrem Anhängsel die Hoffnung verlor. Es war ein Vulkan, der so unerwartet zum Ausbruch kam.

Das ganze Heer der Pygmäen, welche nur Weihrauch u. Lobgesänge für Thiers hatte, diese Geisteszwerge welche sich durch Phrasen bethören lässt, welche die Form bewundern ohne den Inhalt zu begreifen $\mathrm{u}$. die sich gleich anderen Laffen durch die äussern Reize fesseln lassen, schlug ganz wild um sich, als sie durch die Vorgänge des 18 d.M. aus ihrem süssen Taumel gerissen wurde. Und der „kleine grosse Thiers" wie ihn die gesammte Bourgeoispresse nannte, der, weil er dem Fuchsen in der Fabel Lafontaines gleicht, in der Bevölkerung von Paris den Raben zu erblicken glaubte, welchen er durch seine schmeichelnden Reden den Käs, d.h. die Republik aus dem Schnabel zu locken glaubte, wusste nichts Eiligeres zu thun, besonders da sich die Linie mit der Garde Nationale verbündete, als sich aus dem Staube zu machen, u. sich mit der ganzen Bande nach Versailles zu begeben. - Vorläufig ist die Revolution Herr der Situation, die [S. 2] Revolution friedlich durchgefuhrt - wenn auch noch nicht beendigt - von Männern, die weder in der Journalistik, noch auf der Tribüne, weder auf dem Pegasus, noch in der Diplomatie sich einen Namen erworben, weil es grösstentheils Männer sind, die in der Hütte geboren in der Werkstätte ihr Leben fristeten. Das macht's auch was die Bourgeoisie vor Wuth fast bersten macht, das macht es, dass sie sagt wer kennt diese Leute? Sind's Kommunisten, Bonapartisten oder Preussen? Dass dieselbe nicht fragte ob es nicht Orleanisten sind, zeigt mir ihren Pferdefuss, sagt mir, dass ein König ihre stillen Wünsche befriedigen würde.

Gelingt es Morgen Paris wählen zu lassen, haben wir übermorgen die Commune, u. hiemit der Grundstein zum künftigen Arbeiterstaat gelegt. In jedem Falle muss es dahin gebracht werden, denn es gilt die Zukunft unserer Sache, die Frankreichs u. nebenbei gesagt das Leben so mancher Männer, so mancher Ihrer Freunde zu sichern.

Ich will schliessen in der Hoffnung Ihnen geehrtester Bürger bald ausführlicher die Hergänge schildern zu können. Ich will Ihnen nur noch bemerken, dass ich mich hier mit unserm Bundesgenossen Vaillant befinde, welcher mich in seine Nähe weisen liess, um wo möglich meine schwache Hülfe im Interesse"der Sache in Anspruch nehmen zu können.

Seien Sie herzlichst gegrüsst von Ihrem Ergebenen

Leo Frankel

Cher citoyen et ami, J'ai tant à vous dire et je suis pressé que je prefere le re- 
mettre a une autre fois et me contenter aujourd'hui de vous envoyer mes amities cordiales et l'expression de ma bien sincere affection. Des que je le pourrai j'ecrirai aussi à Mme. Wilhelmi notre excellente ami. Bon espoir et cordiale poignée de main.

votre tout devoué ami

Ed. Vaillant

Frankel vous dit sans doute le plus urgent. Rien n'est encore perdu tout peut bien marcher seulement le moment est critique.

Unveränderter Abdruck nach dem Original im IISG, 2 S., $20,5 \times 13,5 \mathrm{~cm}$, Nachlass Becker D I 637

XII

H. BACHRUCH, N. L. PETERSEN, V. SCHILY UND F. A. SORGE

AN JOH. PH. BECKER

Lieber Jean Philippe!

Paris, 29 August 1872

Nochmals die herzliche Aufforderung an dich, sofort die Reise nach dem Haag anzutreten. Um dir es zu erleichtern, setze ich dir hiermit im Verein mit den Freunden Schily u. Bachruch die Summe von 100 frcs. aus, welche du im Haag von mir beziehen wirst.

\section{Brüderlich}

Dein F. A. Sorge

Hier sitzen drei Hallunken

in einer Kneip und trunken

und votiren dir hundert und zehn Cts

zu deiner Reise hinter der Grentz

verlangen aber dass du auf der Hin- oder Rückreise durch Paris passirst

Schily

Lieber Bürger Becker.

Herzlichen Gruss! Hoffe Sie auf Ihrer wahrscheinlichen Durchreise durch Paris persönlich kennen zu lernen.

Henry Bachruch

Im Haag trage ein blaues Band, $u$. folge dem Mann mit dem blauen Band. Die Sitzung findet statt im Saale Concordia, Lombardstraat.

Eine parteigenössische Adresse ist:

Bruno Liebers, Jacob Catsstraat 148

Dein S.[orge]

Ein vierter Hallunke, Petersen erklärt Schreibens- und Unterschreibensunfähig zu sein, und macht sein X Handzeichen, das zu Urkund

Unveränderter Abdruck nach dem Original im IISG, 2 S., $20,5 \times 13.5 \mathrm{~cm}$, Nachlass Becker D II 986 